\title{
Essential Oils and Their Components as Modulators of Antibiotic Activity against Gram-Negative Bacteria
}

\author{
Petruta Aelenei ${ }^{1,2}$, Anca Miron ${ }^{1, *}$, Adriana Trifan ${ }^{1}$, Alexandra Bujor ${ }^{1}$, Elvira Gille ${ }^{3}$ and \\ Ana Clara Aprotosoaie ${ }^{1}$ \\ 1 Department of Pharmacognosy, Faculty of Pharmacy, University of Medicine \\ and Pharmacy Grigore T. Popa-Iasi, Universitatii 16, Iasi 700115, Romania; \\ petruta.aelenei@fitermanpharma.ro (P.A.); adriana.trifan@umfiasi.ro (A.T.); \\ bujoralexandra123@gmail.com (A.B.); anaclara70@yahoo.com (A.C.A.) \\ 2 Regulatory Affairs Department, Fiterman Pharma LLC, Pacurari Road 127, Iasi 700544, Romania \\ 3 Stejarul Biological Research Centre/National Institute of Research and Development for Biological Sciences, \\ Alexandru cel Bun 6, Piatra Neamt 610004, Romania; elgille9@yahoo.com \\ * Correspondence: ancamiron@yahoo.com or anca.miron@umfiasi.ro; Tel.: +40-232-301-600; \\ Fax: +40-232-211-820
}

Academic Editors: Lutfun Nahar, Norazah Basar and Satyajit D. Sarker Received: 30 June 2016; Accepted: 18 July 2016; Published: 28 July 2016

\begin{abstract}
Gram-negative bacteria cause infections that are difficult to treat due to the emergence of multidrug resistance. This review summarizes the current status of the studies investigating the capacity of essential oils and their components to modulate antibiotic activity against Gram-negative bacteria. Synergistic interactions are particularly discussed with reference to possible mechanisms by which essential oil constituents interact with antibiotics. Special emphasis is given to essential oils and volatile compounds that inhibit efflux pumps, thus reversing drug resistance in Gram-negative bacteria. In addition, indifference and antagonism between essential oils/volatile compounds and conventional antibiotics have also been reported. Overall, this literature review reveals that essential oils and their purified components enhance the efficacy of antibiotics against Gram-negative bacteria, being promising candidates for the development of new effective formulations against Gram-negative bacteria.
\end{abstract}

Keywords: essential oils; Gram-negative bacteria; multidrug-resistance; antibiotics; synergistic interactions

\section{Introduction}

Infectious diseases are the second leading cause of death worldwide, being responsible for approximately 15 million deaths each year [1]. This high mortality rate is mainly due to antibiotic resistance. Frequent and improper use of antibiotics favored the emergence and spread of single- and multidrug-resistant microbial strains [2]. In 2009, several European countries reported that $25 \%-50 \%$ of Staphylococcus aureus isolates from patients with nosocomial infections were resistant to methicillin (oxacillin). In 2012, some of these countries (Portugal and Romania) reported percentages higher than $50 \%$ for methicillin-resistant $S$. aureus isolates. Resistance to carbapenems, broad-spectrum $\beta$-lactam antibiotics, has alarmingly increased. From 2009-2012, the percentages of carbapenem-resistant Klebsiella pneumoniae isolates increased dramatically from very low values, such as below $1 \%$ in Romania and $1 \%-5 \%$ in Italy, to values of $10 \%-25 \%$ and $25 \%-50 \%$, respectively [3].

Combination therapy, combining conventional antibiotics and natural products, represents a promising strategy in overcoming antibiotic resistance [4-6]. Such antimicrobial combinations may exhibit synergistic, additive, indifferent or antagonistic effects. Synergy, addition and antagonism occur when the overall effect is greater than, equal to and less than the sum of the individual effects, 
respectively; the absence of any interaction reflects indifference [7,8]. Synergy has potential therapeutic applications, as synergistic combinations have higher efficacy and lower toxicity than their components; due to a multitarget activity, such combinations may prevent the emergence of antibiotic resistance and may be effective against multidrug-resistant microbial strains [7]. Although ignored or rejected in many studies, antagonistic interactions have also important implications, as they might predict a reduction of antimicrobial efficacy [7,9]. Several methodologies have been used to assess antimicrobial interactions between conventional antibiotics and natural products. The most frequently employed is the checkerboard assay, which expresses antimicrobial interactions on the basis of the fractional inhibitory concentration index (FICI) or isobolograms $[7,10]$. There are different interpretations of antimicrobial interactions on the basis of FICI, for example a synergistic interaction is considered to occur if FICI $\leqslant 0.5$ [11-13] or $<1$ [2]. There are also different interpretations of the FICI values denoting additivity $(0.5<\mathrm{FICI} \leqslant 1, \mathrm{FICI}=1)[2,11,12]$, indifference $(1<\mathrm{FICI} \leqslant 2,1<\mathrm{FICI} \leqslant 4,5<$ FICI $\leqslant 4$ ) [11-14] and antagonism (FICI > 1, 2 or 4) [2,11-13].

Most natural products have shown activity predominantly on Gram-positive bacteria [15-17]. Gram-negative bacteria are intrinsically more resistant to antibacterial agents than the Gram-positive ones due to an additional outer membrane acting as an effective barrier for amphipathic agents [18-20] and overexpression of efflux pumps responsible for innate antimicrobial resistance, such as the AcrAB-TolC efflux system [17]. Therefore, the identification of new antimicrobial agents active against Gram-negative bacteria and/or having the ability to modulate the antibiotic susceptibility of Gram-negative bacteria is of considerable importance to therapeutics.

This review summarizes data on essential oils (EOs) and pure volatile compounds that modulate antibiotic activity against Gram-negative bacteria (Acinetobacter baumannii, Escherichia coli, Klebsiella pneumoniae, Pseudomonas aeruginosa, Enterobacter spp., Proteus vulgaris, Salmonella spp.) with special emphasis on synergistic interactions.

\section{Essential Oils and Volatile Compounds Modulating Antibiotic Activity against Acinetobacter baumannii}

Acinetobacter baumannii is an aerobic, pleomorphic, non-motile, non-fermentative, catalase-positive and oxidase-negative coccobacillus [21-23]. It is responsible for skin and soft-tissue infections, being a common Gram-negative bacillus in traumatic injuries (open tibial fractures) and burns [21,24,25]. A. baumannii also causes bacteremia, secondary meningitis, respiratory and urinary tract infections [24], being the fourth among the most frequent etiological agents of hospital-acquired infections after Pseudomonas aeruginosa, Staphylococcus aureus and Klebsiella pneumoniae [22]. Its pathogenicity is due to a high ability to adhere to and form biofilms on both biotic and abiotic surfaces. Outer membrane protein $\mathrm{A}(\mathrm{OmpA})$ and fimbriae play a key role in adhesion, whereas pili and biofilm-associated protein are involved in biofilm formation. In addition, OmpA confers resistance to the serum complement and induces the apoptosis of host cells via mitochondrial dysfunction [21]. Phospholipases are important A. baumannii virulence factors [21,26,27]. Carbapenems (alone or in combination with an aminoglycoside), cephalosporins and $\beta$-lactam- $\beta$-lactamase inhibitor (sulbactam) combinations are commonly used in the treatment of infections caused by antibiotic-susceptible A. baumannii strains. A. baumannii has developed resistance to almost all commercial antimicrobials $(\beta$-lactams, aminoglycosides, quinolones, tetracyclines, glycylcyclines, polymyxins, sulfonamides). Both enzymatic (the production of $\beta$-lactamases, including serine- and metallo- $\beta$-lactamases responsible for carbapenem resistance, aminoglycoside-modifying enzymes) and non-enzymatic mechanisms (alterations in outer membrane proteins, efflux pumps, penicillin-binding proteins, porin channels, DNA gyrase and topoisomerase IV) are responsible for A. baumannii antibiotic resistance [23,28]. Multidrug-resistant (usually resistant to quinolones, cephalosporins and carbapenems), extensively drug-resistant (resistant to almost all commercially available antimicrobials, including cephalosporins, penicillins, carbapenems, aminoglycosides, fluoroquinolones, sulbactam) [29] and pan-resistant (resistant to all standard antimicrobials, except colistin) [28] strains have become a major threat 
to A. baumannii infection control. The treatment options of multidrug-resistant $A$. baumannii infections include polymyxins B and E (colistin), tigecycline and antibiotic combinations (colistin or imipenem and rifampin). Polymyxins cause severe side effects, such as nephrotoxicity and bronchoconstriction. Besides, A. baumannii isolates lacking susceptibility to tigecycline have been detected [28].

Both EOs and volatile compounds have been reported to modulate the antibiotic susceptibility of A. baumannii (Table 1). Coriander (Coriandrum sativum L., Apiaceae) EO acted synergistically with conventional antibiotics against two chloramphenicol-resistant $A$. baumannii reference strains (LMG 1025, LMG 1041). Coriander EO significantly increased the susceptibility to chloramphenicol of both strains as indicated by the decrease of minimum inhibitory concentration (MIC) values of chloramphenicol in combination (16- and 31-fold decrease against A. baumannii LMG 1025 and LMG 1041, respectively) [30]. Coriander EO has been reported to increase the permeability of bacterial membrane, thus impairing membrane potential, respiratory activity and efflux pump activity [31]. EOs are active against both Gram-positive and Gram-negative bacteria [8,32,33]. Coriander EO showed higher bactericidal effects against A. baumannii and other Gram-negative bacteria (Escherichia coli, Klebsiella pneumoniae, Salmonella typhimurium) compared to Gram-positive ones (Bacillus cereus, Staphylococcus aureus, Enterococcus faecalis). These differences in activity were attributed to the ability of S-(+)-linalool (Figure 1), the major constituent of coriander EO, to disrupt the negatively-charged outer membrane of Gram-negative bacteria, on the one hand, and to decrease the cell wall thickness of Gram-positive bacteria with a consequent increase in resistance to solvents and dyes, on the other hand [31]. Multidrug-resistant A. baumannii is an etiological agent of traumatic and burn wound infections. In these infections, hemorrhagic bullae and necrosis facilitate the entry of the pathogen into the bloodstream, leading to complications (bacteremia, septicemia). Therefore, multidrug-resistant $A$. baumannii is a major threat for patients with traumatic and burn injuries [21]. Linalool-rich EOs, such as rosewood (Aniba rosaeodora Ducke, Lauraceae) and myrtle (Myrtus communis L., Myrtaceae) EOs (60.1\% and 18.32\%-26.59\% linalool, respectively), acted synergistically with conventional antibiotics (gentamicin, ciprofloxacin, polymyxin B) against A. baumannii reference strains and multidrug-resistant wound isolates [24,34]. Synergism between linalool-rich EOs and conventional antibiotics may be attributed, at least in part, to the membrane permeabilization activity of EOs, resulting in an enhanced penetration of antibiotics inside the cell [24]. Except polymyxin B, which affects the membrane structure, the other antibiotics, showing synergy in combination with linalool-rich EOs, act on different targets: chloramphenicol and gentamicin impair protein synthesis, while ciprofloxacin inhibits topoisomerase II (DNA gyrase) and IV and, consequently, DNA replication [10]. As myrtle EOs and polymyxin B target the same site, the membrane, their combination was efficient in increasing the sensitivity of multidrug-resistant $A$. baumannii isolates. Myrtle EOs and ciprofloxacin have different target sites (membrane and DNA, respectively), and therefore, their combination caused the most frequent synergistic effects [24]. Two red river gum (Eucalyptus camaldulensis Dehnh., Myrtaceae) EOs showed in vitro synergistic activity with polymyxin B against 22 A. baumannii strains (two standard strains and 20 multidrug-resistant wound isolates). Oxygenated sesquiterpene spathulenol (18.9\%) and monoterpene alcohols 1,8-cineole (eucalyptol) $(7.62 \%)$ and terpinen-4-ol (7.59\%) (Figure 1) are mainly responsible for the red river gum EOs' antibacterial activity. Polymyxin B-EOs combinations increased bacterial sensitivity by similar mechanisms of activity, as both act by affecting the cell membrane structures [25,35]. Both rosewood (Aniba rosaeodora Ducke, Lauraceae) and geranium (Pelargonium graveolens L., Geraniaceae) EOs (60.1\% linalool and $47.3 \%$ citronellol, respectively) acted synergistically with gentamicin against A. baumannii ATCC 19606. The strong synergism could be favored by the two major terpenalcohols, linalool and citronellol [34]. It has been reported that such compounds are able to compromise the integrity of the cytoplasmic membrane, alter the physicochemical surface properties and determine $\mathrm{K}^{+}$leakage [36]; thus, they disrupt the bacterial membrane and increase the intracellular penetration of gentamicin, a protein synthesis inhibitor [10,34,36]. Patients requiring dialysis, catheterization and mechanical ventilation, but also immunocompromised patients have a high risk of developing A. baumannii 
infections [21,22]. Immortelle (Helichrysum italicum (Roth) G. Don., Asteraceae) EO reduced the resistance to chloramphenicol of A. baumannii ATCC 19606 and the AB1 strain isolated from central venous catheters of critical care patients $[37,38]$. In the case of both strains, immortelle EO reduced by eight-fold the MIC value of chloramphenicol in combination, whereas phenylalanine-arginine $\beta$-naphthylamide $(\mathrm{Pa} \beta \mathrm{N})$, a potent efflux pumps inhibitor, caused only a two-fold reduction. Geraniol (Figure 1), a component of immortelle EO, is an inhibitor of bacterial efflux pumps, which are responsible for both antibiotic resistance and resistance to host innate immune defense [37,39]. Cinnamon (Cinnamomum zeylanicum Nees., Lauraceae) and lemon (Citrus limon L. Burm. F., Rutaceae) EOs showed strong synergistic effects in combination with amikacin against a multidrug-resistant A. baumannii clinical isolate (A-06 strain) [40]. The study provides no information on the chemical composition of both cinnamon and lemon EOs. However, a permeabilization of the bacterial membrane by certain EO constituents followed by an increased penetration of amikacin might explain, at least in part, these synergistic effects.

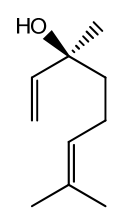

S-(+)-linalool

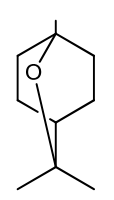

1,8-cineole (eucalyptol)<smiles>CC1=CCC(O)(C(C)C)CC1</smiles>

terpinen-4-ol<smiles>CC(C)=CCCC(C)=CCO</smiles>

geraniol<smiles>Cc1ccc(C(C)C)c(O)c1</smiles>

thymol

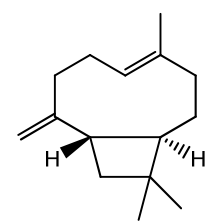

$\beta$-caryophyllene<smiles>Cc1ccc(C(C)C)cc1O</smiles>

carvacrol<smiles>C=C1CCC2C(C3C1CCC3(C)O)C2(C)C</smiles>

spathulenol

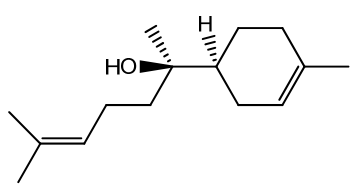<smiles>C=CCc1ccc(O)c(OC)c1</smiles><smiles>C/C=C/c1ccc(OC)cc1</smiles>

$\alpha$-bisabolol<smiles>C=C[C@]1(C)CC[C@@H](C(=C)C)C[C@H]1C(=C)C</smiles>

$\beta$-elemene<smiles>C[C@H]1CCC2C1C1C(CC[C@@]2(C)O)C1(C)C</smiles>

viridiflorol

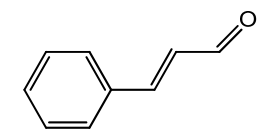

trans-cinnamaldehyde

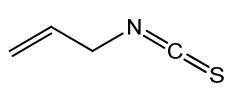

allyl isothiocyanate

Figure 1. Essential oil components acting synergistically with conventional antibiotics against Gram-negative bacteria. 
Table 1. Essential oils (EOs) and volatile compounds modulating the antibiotic activity against Acinetobacter baumannii.

\begin{tabular}{|c|c|c|c|c|c|c|c|}
\hline $\begin{array}{c}\text { Essential Oil/ } \\
\text { Volatile Compound }\end{array}$ & Vegetal Source & Major Constituent & $\begin{array}{c}\text { Acinetobacter baumannii } \\
\text { Strain }\end{array}$ & Antibiotic & Method & Effect & Ref. \\
\hline Tea tree EO & $\begin{array}{c}\text { Melaleucae folium } \\
\text { (Melaleuca alternifolia (Maiden. and } \\
\text { Beach.) Cheel., Myrtaceae, Tea tree) }\end{array}$ & Terpinen-4-ol (30.3\%) & ATCC 19606 & Gentamicin & $\mathrm{CB}(\mathrm{FICI}=0.5)$ & $\mathrm{S}$ & [34] \\
\hline \multirow{3}{*}{ Myrtle EO } & \multirow{3}{*}{$\begin{array}{c}\text { Myrti folium (Myrtus communis L., } \\
\text { Myrtaceae, Myrtle) }\end{array}$} & $\begin{array}{c}\text { Linalool }(22.276 \%) \\
\text { Myrtenyl acetate }(16.561 \%) \\
1,8 \text {-Cineole }(13.250 \%)\end{array}$ & \multirow{3}{*}{$\begin{array}{c}\text { ATCC } 19606 \text { ATCC } \\
\text { BAA747 and } 22 \text { clinical } \\
\text { isolates of which MDR } \\
\text { strains: Aba-6637, } \\
\text { Aba-4914, Aba-5055 }\end{array}$} & \multirow{2}{*}{ Ciprofloxacin } & \multirow{3}{*}{$\begin{array}{c}\mathrm{CB}(\mathrm{FICI}<0.5) \\
\mathrm{TK}\end{array}$} & \multirow[t]{2}{*}{$\mathrm{S}$} & \multirow{3}{*}{ [24] } \\
\hline & & $\begin{array}{c}\text { Linalool }(18.320 \%) \\
\text { Myrtenyl acetate }(18.009 \%) \\
1.8 \text {-Cineole }(16.878 \%)\end{array}$ & & & & & \\
\hline & & $\begin{array}{c}\text { Linalool }(26.591 \%) \\
\text { Myrtenyl acetate }(18.489 \%) \\
\text { 1,8-Cineole }(15.762 \%)\end{array}$ & & Polymyxin B & & $\mathrm{S}$ & \\
\hline \multirow{5}{*}{ Longbeak eucalyptus EO } & \multirow{5}{*}{$\begin{array}{l}\text { Eucalypti folium (Eucalyptus } \\
\text { camaldulensis Dehnh., Myrtaceae, } \\
\text { Longbeak eucalyptus) }\end{array}$} & \multirow{3}{*}{ Spathulenol (18.90\%) } & $\begin{array}{l}\text { Aba- } 4914 \\
\text { Aba-5055 }\end{array}$ & \multirow[t]{2}{*}{ Ciprofloxacin } & $\mathrm{CB}(\mathrm{FICI}<0.5)$ & $S$ & \multirow{5}{*}[25]{} \\
\hline & & & Aba- 6673 & & $\mathrm{CB}(\mathrm{FICI}=0.53)$ & $\mathrm{Ad}$ & \\
\hline & & & $\begin{array}{l}\text { Aba- } 4914 \\
\text { Aba- } 6673\end{array}$ & Gentamicin & $\mathrm{CB}(\mathrm{FICI}<0.5)$ & $\mathrm{s}$ & \\
\hline & & \multirow{2}{*}{ Spathulenol (21.39\%) } & & & $\mathrm{CB}(\mathrm{FICI}=0.5)$ & Ad & \\
\hline & & & $\begin{array}{l}\text { ATCC } 19606 \\
\text { Aba-4914 } \\
\text { Aba-5055 }\end{array}$ & Polymyxin B & $\begin{array}{c}\mathrm{CB}(\mathrm{FICI}<0.5) \\
\text { TK }\end{array}$ & $\mathrm{S}$ & \\
\hline Rosewood EO & $\begin{array}{l}\text { Anibae lignum (Aniba rosaeodora } \\
\text { Ducke, Lauraceae, Rosewood) }\end{array}$ & Linalool $(60.1 \%)$ & ATCC 19606 & Gentamicin & $\mathrm{CB}(\mathrm{FICI}=0.11)$ & $S$ & [34] \\
\hline \multirow{4}{*}{ Cinnamon EO } & \multirow{4}{*}{$\begin{array}{c}\text { Cinnamomi cortex } \\
\text { (Cinnamomum zeylanicum Nees, } \\
\text { Lauraceae, Cinnamon) }\end{array}$} & \multirow{4}{*}{ - } & \multirow{4}{*}{ Clinical isolate A-06 } & Amikacin & $\mathrm{CB}(\mathrm{FICI}=0.045)$ & $\mathrm{S}$ & \multirow{4}{*}{ [40] } \\
\hline & & & & Gentamicin & $\mathrm{CB}(\mathrm{FICI}=0.5)$ & $\mathrm{Ad}$ & \\
\hline & & & & Imipenem & $\mathrm{CB}(\mathrm{FICI}=2)$ & I & \\
\hline & & & & Meropenem & $\mathrm{CB}(\mathrm{FICI}=1.5)$ & I & \\
\hline
\end{tabular}


Table 1. Cont.

\begin{tabular}{|c|c|c|c|c|c|c|c|}
\hline $\begin{array}{c}\text { Essential Oil/ } \\
\text { Volatile Compound }\end{array}$ & Vegetal Source & Major Constituent & $\begin{array}{c}\text { Acinetobacter } \\
\text { baumannii Strain }\end{array}$ & Antibiotic & Method & Effect & Ref. \\
\hline \multirow{12}{*}{ Coriander EO } & \multirow{12}{*}{$\begin{array}{l}\text { Coriandri fructus (Coriandrum sativum } \\
\text { L., Apiaceae, Coriander) }\end{array}$} & \multirow{12}{*}{-} & \multirow{6}{*}{ LMG 1025} & Cefoperazone & $\mathrm{CB}(\mathrm{FICI}=0.750)$ & Ad & \multirow{12}{*}[30]{} \\
\hline & & & & Chloramphenicol & $\mathrm{CB}(\mathrm{FICI}=0.312)$ & $\mathrm{S}$ & \\
\hline & & & & Ciprofloxacin & $\mathrm{CB}(\mathrm{FICI}=0.281)$ & $\mathrm{S}$ & \\
\hline & & & & Gentamicin & $\mathrm{CB}(\mathrm{FICI}=0.250)$ & $\mathrm{S}$ & \\
\hline & & & & Piperacillin & $\mathrm{CB}(\mathrm{FICI}=1)$ & $\mathrm{Ad}$ & \\
\hline & & & & Tetracycline & $\mathrm{CB}(\mathrm{FICI}=0.312)$ & $\mathrm{S}$ & \\
\hline & & & \multirow{6}{*}{ LMG 1041} & Cefoperazone & $\mathrm{CB}(\mathrm{FICI}=1)$ & $\mathrm{Ad}$ & \\
\hline & & & & Chloramphenicol & $\mathrm{CB}(\mathrm{FICI}=0.047)$ & $\mathrm{S}$ & \\
\hline & & & & Ciprofloxacin & $\mathrm{CB}(\mathrm{FICI}=0.375)$ & $\mathrm{S}$ & \\
\hline & & & & Gentamicin & $\mathrm{CB}(\mathrm{FICI}=0.375)$ & $\mathrm{S}$ & \\
\hline & & & & Piperacillin & $\mathrm{CB}(\mathrm{FICI}=0.625)$ & $\mathrm{Ad}$ & \\
\hline & & & & Tetracycline & $\mathrm{CB}(\mathrm{FICI}=0.185)$ & $\mathrm{S}$ & \\
\hline Immortelle EO & $\begin{array}{l}\text { Helichrysi flos (Helichrysum italicum } \\
\text { (Roth) G. Don, } \\
\text { Asteraceae, Immortelle) }\end{array}$ & - & $\begin{array}{l}\text { ATCC } 19606 \\
\text { Clinical isolate } \\
\text { AB1 }\end{array}$ & Chloramphenicol & $\begin{array}{l}\text { FR (8-fold } \\
\text { reduction in MIC) }\end{array}$ & S & [37] \\
\hline Oregano EO & $\begin{array}{l}\text { Origani herba (Origanum vulgare } \\
\text { Linn., Lamiaceae, Oregano) }\end{array}$ & Cymenol (58.6\%) & ATCC 19606 & Gentamicin & $\mathrm{CB}(\mathrm{FICI}=0.65)$ & $\mathrm{Ad}$ & [34] \\
\hline Geranium EO & $\begin{array}{c}\text { Pelargonii herba } \\
\text { (Pelargonium graveolens L., } \\
\text { Geraniaceae, Geranium) }\end{array}$ & Citronellol (47.3\%) & ATCC 19606 & Gentamicin & $\mathrm{CB}(\mathrm{FICI}=0.11)$ & S & \\
\hline \multirow{4}{*}{ Lemon EO } & \multirow{4}{*}{$\begin{array}{l}\text { Citri pericarpium (Citrus limon L. } \\
\text { Burm. F., Rutaceae, Lemon) }\end{array}$} & \multirow{4}{*}{-} & \multirow{4}{*}{$\begin{array}{l}\text { Clinical isolate } \\
\text { A-06 }\end{array}$} & Amikacin & $\mathrm{CB}(\mathrm{FICI}=0.037)$ & $\mathrm{S}$ & \multirow{4}{*}{ [40] } \\
\hline & & & & Gentamicin & $\mathrm{CB}(\mathrm{FICI}=0.5)$ & $\mathrm{Ad}$ & \\
\hline & & & & Imipenem & $\mathrm{CB}(\mathrm{FICI}=2)$ & I & \\
\hline & & & & Meropenem & $\mathrm{CB}(\mathrm{FICI}=2)$ & I & \\
\hline
\end{tabular}

Ad: addition; S: synergy; I: indifference; CB: checkerboard assay; TK: time kill assay; FICI: fractional inhibitory concentration index; FR: fold reduction in MIC; MIC: minimum inhibitory concentration; MDR: multidrug-resistant. 


\section{Essential Oils and Volatile Compounds Modulating Antibiotic Activity against Escherichia coli}

Escherichia coli is a facultative anaerobic, non-spore-forming Gram-negative bacterium, commonly abundant in the normal intestinal tract flora. Pathogenic strains are spread mainly by contaminated food and water being responsible for a wide range of infections. Identification of $\mathrm{O}$ (lipopolysaccharide) and $\mathrm{H}$ (flagellar) antigens allowed the classification of enteric $E$. coli into seven pathovars responsible for certain diseases: diarrhea in children (enteropathogenic, diffusely adherent and enteroaggregative E. coli), hemolytic-uremic syndrome (enterohemorrhagic E. coli), traveler's diarrhea (enterotoxigenic E. coli), dysentery (enteroinvasive E. coli) and Crohn's disease (adherent invasive E. coli) [41,42]. There are also two pathovars of the extraintestinal type, which are involved in urinary tract infections (uropathogenic E. coli) and meningitis in newborns (neonatal meningitis E. coli) [43]. Cell adhesion through pili or fimbriae is characteristic for all pathovars, except for enteroinvasive E. coli Besides, specific virulence factors have been identified for different pathovars, such as hemolysin (uropathogenic and enteroinvasive E. coli) [44,45], Shiga toxins (enterohemorrhagic and enteroinvasive E. coli) [45] and heat-labile and heat-stable toxins (enterotoxigenic E. coli) [46]. E. coli is intrinsically resistant to penicillin $G$ due to the outer membrane barrier, but extended spectrum $\beta$-lactamase production is responsible for the resistance to a broad spectrum of $\beta$-lactams, including penicillins, cephalosporins (third- and fourth-generation cephalosporins) and monobactams [47]. Carbapenem resistance of E. coli is also a therapeutic challenge because many nosocomial isolates have been reported to synthesize carbapenemases [48]. The alteration of quinolone targets (DNA gyrase, topoisomerase IV), the decrease in membrane permeability and the overexpression of efflux pumps explain the resistance of $E$. coli to quinolones [49]. The production of acetyltransferases, nucleotidyltransferases, phosphotransferases and methylation of $16 \mathrm{~S}$ rRNA are responsible for the resistance to aminoglycosides [42]. Treatment options of infections caused by extended spectrum $\beta$-lactamase-producing E. coli include imipenem, meropenem and third-generation cephalosporins in combination with an enzyme inhibitor (clavulanic acid) [50].

A feasible approach to reduce the antibiotic resistance of $E$. coli is the combination of conventional antibiotics with EOs or volatile compounds (Table 2). A strong synergy was reported for two Moroccan thyme (Thymus maroccanus L. and Thymus broussonetii L., Lamiaceae) EOs in combination with chloramphenicol against E. coli strains: AG100 (wild-type E. coli K-12) and AG102 (AG100 derivative overexpressing AcrAB pump responsible for resistance to tetracycline, chloramphenicol, ampicillin, nalidixic acid and rifampicin) [51,52]. Both EOs decreased the MIC values of chloramphenicol against E. coli AG100 and AG102 strains by four- and 32-fold, respectively. The synergy could be attributed to the alteration of efflux pump activity by certain EO constituents, but also to the ability of carvacrol $(76.35 \%$ in T. maroccanus $\mathrm{EO}, 39.77 \%$ in $\mathrm{T}$. broussonetii $\mathrm{EO}$ ) to cause the permeabilization and depolarization of the E. coli cytoplasmic membrane, thus facilitating the passage of the antibiotic to the bacterial cell [52,53]. Carvacrol (Figure 1) is a well-known antibacterial agent [54]; in the case of Gram-negative bacteria, carvacrol disintegrates the polysaccharidic capsule, followed by an increase in the fluidity and permeability of the cytoplasmic membrane [55]. In addition, it acts as a proton exchanger, thus affecting the cytoplasmic $\mathrm{pH}$ and proton motive force with a subsequent reduction of ATP and DNA synthesis and inhibition of cytoplasmic enzymes, leading to bacterial cell death [56,57]. Similar results were obtained for another Moroccan thyme species (T. riatarum L., Lamiaceae) EO in combination with chloramphenicol against E. coli AG100 and AG100A (AG100 derivative with AcrAB pump deleted, but resistant to kanamycin). T. riatarum $\mathrm{EO}$ decreased the MIC values of chloramphenicol against AG100 and AG100A by four- and two-fold, respectively; the synergy could be attributed to efflux pump blocking activity of $T$. riatarum $\mathrm{EO}$, thus increasing the activity of chloramphenicol [58]. The lemon thyme (Thymus pulegioides L., Lamiaceae) EO showed synergistic effects with tetracycline and chloramphenicol against E. coli ATCC 25922. Surprisingly, the combination between lemon thyme EO and streptomycin displayed an antagonistic effect related to a possible competition for the same target: the bacterial 30S ribosomal subunit; binding of this subunit leads to inhibition of protein synthesis. Lemon thyme EO contained geraniol (66.59\%) as a major constituent. Geraniol has also been reported in immortelle (Helichrysum italicum (Roth) G. Don, Asteraceae) EO [59]. Immortelle 


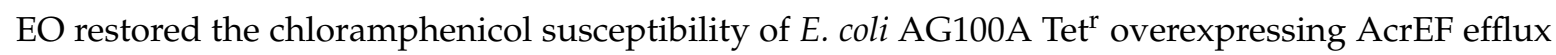
pumps, responsible for tetracycline resistance. The MIC value of chloramphenicol was decreased by 256 -fold in combination with immortelle EO, suggesting the ability of the latter to inhibit AcrEF efflux pumps [37]. Other oxygenated monoterpene-rich EOs, such as savory (Satureja kitaibelii Wierzb. ex Heuff., Lamiaceae), rosewood (Aniba rosaeodora Ducke, Lauraceae), geranium (Pelargonium graveolens L., Geraniaceae) and tea tree (Melaleuca alternifolia (Maiden and Beach) Cheel., Myrtaceae) EOs exhibited synergistic effects in combination with different antibiotics (chloramphenicol, tetracycline, gentamicin) against E. coli ATCC 25922 [34,60]. Synergistic effects might be correlated with the damage of the microbial membrane lipids by terpene alcohols [34] followed by an increased penetration of antibiotics into the bacterial cell. Modulatory effects of rosemary (Rosmarinus officinalis L., Lamiaceae) EO on the E. coli EC27 clinical isolate resistant to aminoglycosides have been investigated. The antimicrobial activities of amikacin, neomycin and gentamycin were increased by rosemary EO [61]. 1,8-Cineol (eucalyptol) (Figure 1), the main component of rosemary EO, has been reported to disintegrate the outer membrane and reduce the nucleoplasm [39]. On the other hand, all three aminoglycosides bind to the $30 \mathrm{~S}$ ribosomal subunit, blocking protein synthesis [10]. Modulation of antibiotic activity by EOs through gaseous contact is a new approach that should be taken into consideration especially, in the case of respiratory infections. Cidreira-do-mato [62], also known as cidreira-brava [63] (Hyptis martiusii Benth., Lamiaceae), EO increased the susceptibility to tobramycin of E. coli ATCC 25922 and the EC27 multidrug-resistant surgical wound isolate by $76.5 \%$ and $80 \%$, respectively; in addition, it considerably reduced the resistance to gentamicin and amikacin of the EC27 isolate (by $40 \%$ and $82.4 \%$, respectively). The main constituents of cidreira-brava EO were bicyclogermacrene $(10.6 \%)$, trans-caryophyllene (9.2\%), caryophyllene oxide (7.4\%), 1,8-cineole (7\%) and $\delta$-2-carene (6.8\%) [64]. Neither for cidreira-brava EO, nor for its main constituents studies were performed in order to assess the mechanisms of synergism with aminoglycosides, but the permeabilization of the bacterial membrane by EO components might be partially responsible for the increase in antibiotic activity.

Several studies have reported interactions between EO constituents and conventional antibiotics against E. coli strains. Gallucci et al. [65] detected a potent synergism between eugenol/thymol and penicillin against $E$. coli resistant to $\beta$-lactam antibiotics. Thymol (Figure 1) also acted synergistically with penicillin against multidrug-resistant $E$. coli N00 666 [66]. These synergisms might be attributed to the ability of terpene phenols to increase the antibiotic permeation into the bacterial cell. Both thymol and carvacrol showed more synergistic interactions with antibiotics against $E$. coli strains in comparison with eugenol [66]. This difference in synergistic potential is mainly due to different electron delocalization. In the case of thymol and carvacrol, the delocalized electrons enable the release of the proton belonging to the phenolic hydroxyl group. The released proton acts as a proton exchanger and reduces the $\mathrm{pH}$ gradient across the cytoplasmic membrane, thus decreasing the bacterial membrane potential and causing ATP depletion [56]. Eugenol (Figure 1) has been reported to target both the bacterial membrane, leading to a non-specific increase in antibiotic penetration, and different metabolic pathways [18]. However, in the case of eugenol, the presence of the methoxyl group impairs the release of the phenolic hydroxyl proton. This structural pattern might explain not only the weaker antimicrobial activity of eugenol in comparison with thymol and carvacrol [67], but also the reduced number of synergisms between eugenol and conventional antibiotics. Cinnamaldehyde (Figure 1), a volatile phenylpropanoid, increased the antibiotic susceptibility of E. coli N00 666 [66]. The effect could be attributed, at least in part, to the ability of cinnamaldehyde to inhibit ATP-ase activity with a consequent alteration in bacterial membrane permeability $[10,68]$. An antagonistic interaction against $E$. coli has been reported for myrcene-penicillin combination [65]. Myrcene is a monoterpene present in different percentages in EOs isolated from hemp (Cannabis sativa L., Cannabaceae) (21\%-35\%) [69], hop (Humulus lupulus L., Cannabaceae) (6\%-10\%) [70], chameleon plant/Chinese lizard tail/fishwort/heartleaf (Houttuynia cordata Thunb., Saururaceae) (up to 30.8\%) [71,72] and lemon grass (Cymbopogon citratus (DC.) Stapf., Poaceae) (3\%-8\%) [73]. The antagonism might be attributed to a competition between myrcene and penicillin for the same targets, namely penicillin-binding proteins [10]. 
Table 2. Essential oils and volatile compounds modulating the antibiotic activity against Escherichia coli.

\begin{tabular}{|c|c|c|c|c|c|c|c|}
\hline $\begin{array}{c}\text { Essential Oil/ } \\
\text { Volatile Compound }\end{array}$ & Vegetal Source & Major Constituent & Escherichia coli Strain & Antibiotic & Method & Effect & Ref. \\
\hline Oregano EO & $\begin{array}{l}\text { Origani herba (Origanum vulgare Linn., } \\
\text { Lamiaceae, Oregano) }\end{array}$ & Cymenol (58.6\%) & ATCC 25922 & Gentamicin & $\mathrm{CB}(\mathrm{FICI}=0.65)$ & Ad & [34] \\
\hline \multirow{4}{*}{ Thyme EO } & \multirow{2}{*}{$\begin{array}{l}\text { Thymi herba (Thymus maroccanus L., } \\
\text { Lamiaceae, Za'ater/Azukeni) }\end{array}$} & \multirow{2}{*}{ Carvacrol (76.35\%) } & AG100 & \multirow{4}{*}{ Chloramphenicol } & FR (4-fold reduction in MIC) & \multirow{4}{*}{$\mathrm{s}$} & \multirow{4}{*}{52} \\
\hline & & & AG102 & & FR (32-fold reduction in MIC) & & \\
\hline & \multirow{2}{*}{$\begin{array}{l}\text { Thymi herba (Thymus broussonetii L., } \\
\text { Lamiaceae, Za'atar Essaouiri) }\end{array}$} & \multirow{2}{*}{$\begin{array}{l}\text { Carvacrol }(39.77 \%) \\
\text { Borneol }(12.03 \%)\end{array}$} & AG100 & & FR (4-fold reduction in MIC) & & \\
\hline & & & AG102 & & FR (32-fold reduction in MIC) & & \\
\hline \multirow{5}{*}{ Lemon thyme EO } & \multirow{5}{*}{$\begin{array}{l}\text { Thymi herba (Thymus pulegioides L., } \\
\text { Lamiaceae, Lemon } \\
\text { thyme/broad-leaved thyme) }\end{array}$} & \multirow{5}{*}{ Geraniol (66.59\%) } & \multirow{5}{*}{ ATCC 25922} & \multirow{2}{*}{ Tetracycline } & $\mathrm{CB}(\mathrm{FICI}=0.43-0.76)$ & $\mathrm{s}$ & \multirow{5}{*}{74} \\
\hline & & & & & CB (FICI $=0.98-1.09)$ & $\mathrm{Ad}$ & \\
\hline & & & & Streptomycin & $\mathrm{CB}(\mathrm{FICI}=1.20-2.80)$ & A & \\
\hline & & & & \multirow{2}{*}{ Chloramphenicol } & $\mathrm{CB}(\mathrm{FICI}=0.21-0.87)$ & $\mathrm{s}$ & \\
\hline & & & & & $\mathrm{CB}(\mathrm{FICI}=0.98-1.09)$ & $\mathrm{Ad}$ & \\
\hline \multirow{4}{*}{ Savory EO } & \multirow{4}{*}{$\begin{array}{c}\text { Saturejae herba (Satureja kitaibelii } \\
\text { Wierzb. ex Heuff., } \\
\text { Lamiaceae, Savory) }\end{array}$} & \multirow{4}{*}{ Geraniol (50.4\%) } & \multirow{4}{*}{ ATCC 25922} & \multirow{2}{*}{ Chloramphenicol } & $\mathrm{CB}(\mathrm{FICI}=0.21-0.87)$ & $\mathrm{s}$ & \multirow{4}{*}{60} \\
\hline & & & & & $\mathrm{CB}(\mathrm{FICI}=0.98-1.09)$ & $\mathrm{Ad}$ & \\
\hline & & & & \multirow{2}{*}{ Tetracycline } & $\mathrm{CB}(\mathrm{FICI}=0.32-0.87)$ & $\mathrm{s}$ & \\
\hline & & & & & $\mathrm{CB}(\mathrm{FICI}=0.98-1.09)$ & $\mathrm{Ad}$ & \\
\hline Sandarac EO & $\begin{array}{l}\text { Tetraclinis coni (Tetraclinis articulata } \\
\text { (Vahl.) Masters., } \\
\text { Cupressaceae, Sandarac) }\end{array}$ & $\begin{array}{c}\alpha \text {-Campholenal }(16.34 \%) \\
\text { Trans-Pinocarveol }(15.45 \%) \\
\text { Verbenone }(13.36 \%) \\
\text { Cis-Verbenol }(12.36 \%)\end{array}$ & ATCC 10536 & Amoxicillin & $\mathrm{CB}(\mathrm{FICI}=1)$ & Ad & [75] \\
\hline \multirow{3}{*}{ Rosemary EO } & \multirow{3}{*}{$\begin{array}{l}\text { Rosmarini folium (Rosmarinus } \\
\text { officinalis L., Lamiaceae, Rosemary) }\end{array}$} & \multirow{3}{*}{1,8 -Cineole $(30.87 \%)$} & \multirow{3}{*}{ Clinical isolate } & Amikacin & FR ( 250-fold reduction in MIC) & \multirow{3}{*}{$\mathrm{s}$} & \multirow{3}{*}{61} \\
\hline & & & & Neomycin & FR ( 250-fold reduction in MIC) & & \\
\hline & & & & Gentamicin & $\begin{array}{l}\text { FR ( } \sim 4200 \text {-fold reduction in } \\
\text { MIC) }\end{array}$ & & \\
\hline \multirow{7}{*}{$\begin{array}{l}\text { Cidreira-do-mato/ } \\
\text { cidreira-brava EO }\end{array}$} & & & & Amikacin & FR $(-)$ & I & \\
\hline & Huntic folium (Huntic martiusii Benth & Bicyclogermacrene $(10.6 \%)$ & & & $\operatorname{IZGC}(+2.4 \%)$ & $\mathrm{s}$ & \\
\hline & $\begin{array}{l}\text { Lamiaceae, Cidreira-do-mato/ } \\
\text { Lira }\end{array}$ & $\begin{array}{l}\text { Trans-Caryophyllene }(9.2 \%) \\
\text { Caryophyllene oxide }(7.4 \%)\end{array}$ & ATCC 25922 & Gentamycin & FR $(-)$ & I & [64] \\
\hline & cidreira-brava) & 1,8-Cineole $(7 \%)$ & & & IZGC $(+17.6 \%)$ & $\mathrm{s}$ & \\
\hline & & & & Neomycin & FR (4-fold reduction in MIC) & $\mathrm{s}$ & \\
\hline & & & & Kanamycin & FR (-) & I & \\
\hline & & & & Tobramycin & IZGC (+76.5\%) & $\mathrm{s}$ & \\
\hline & & & & Amikacin & FR (-) & I & \\
\hline & Huntis folium (Huntis martiusii Benth & Bicyclogermacrene $(10.6 \%)$ & & & IZGC $(+82.4 \%)$ & $\mathrm{s}$ & \\
\hline Cidreira-do-mato/ & $\begin{array}{l}\text { Lamiaceae, } \\
\text { Lest }\end{array}$ & Trans-Caryophyllene $(9.2 \%)$ & Clinical isolate & Gentamycin & FR (-) & I & [64] \\
\hline & Cidreira-do-mato/cidreira-brava) & 1,8 -Cineole $(7 \%)$ & & & $\operatorname{IZGC}(+40.0 \%)$ & $\mathrm{S}$ & \\
\hline & & & & Neomycin & FR (-) & I & \\
\hline & & & & Kanamycin & FR (-) & I & \\
\hline & & & & Tobramycin & IZGC (+80.0\%) & $\mathrm{s}$ & \\
\hline
\end{tabular}


Table 2. Cont

\begin{tabular}{|c|c|c|c|c|c|c|c|}
\hline $\begin{array}{c}\text { Essential Oil/ } \\
\text { Volatile Compound }\end{array}$ & Vegetal Source & Major Constituent & Escherichia coli Strain & Antibiotic & Method & Effect & Ref. \\
\hline \multirow{2}{*}{ Thyme EO } & \multirow{2}{*}{$\begin{array}{c}\text { Thymi herba (Thymus riatarum } \\
\text { Humbert and Maire., Lamiaceae, } \\
\text { Moroccan thyme) }\end{array}$} & \multirow{2}{*}{ Borneol (41.67\%) } & AG100 & \multirow{2}{*}{ Chloramphenicol } & FR (4-fold reduction in MIC) & \multirow{2}{*}{ S } & \multirow{2}{*}{58} \\
\hline & & & AG100A & & FR (2-fold reduction in MIC) & & \\
\hline \multirow{3}{*}{ Thyme EO } & \multirow{3}{*}{$\begin{array}{l}\text { Thymi herba (Thymus saturejoides } \\
\text { Coss., Lamiaceae, Azoukni) }\end{array}$} & $\begin{array}{l}\text { Carvacrol }(25.3 \%) \\
\text { Borneol }(19.7 \%)\end{array}$ & \multirow{3}{*}{ ATCC 25922} & \multirow{3}{*}{ Cefixime } & \multirow{3}{*}{$\mathrm{CB}(\mathrm{FICI}=1.25)$} & \multirow{3}{*}{ I } & \multirow{3}{*}{76} \\
\hline & & $\begin{array}{l}\text { Carvacrol }(26.5 \%) \\
\text { Borneol }(20.1 \%)\end{array}$ & & & & & \\
\hline & & $\begin{array}{c}\text { Carvacrol }(45.3 \%) \\
\text { Borneol }(7.5 \%)\end{array}$ & & & & & \\
\hline Rosewood EO & $\begin{array}{l}\text { Anibae lignum (Aniba rosaeodora } \\
\text { Ducke, Lauraceae, Rosewood) }\end{array}$ & Linalool $(60.1 \%)$ & ATCC 25922 & Gentamicin & $\mathrm{CB}(\mathrm{FICI}=0.35)$ & $\mathrm{s}$ & [34] \\
\hline Geranium EO & $\begin{array}{c}\text { Pelargonii herba (Pelargonium } \\
\text { graveolens L'Hér., } \\
\text { Geraniaceae, Geranium) }\end{array}$ & Citronellol (47.3\%) & ATCC 25922 & Gentamicin & $\mathrm{CB}(\mathrm{FICI}=0.30)$ & $S$ & [34] \\
\hline Tea tree EO & $\begin{array}{l}\text { Melaleucae folium (Melaleuca } \\
\text { alternifolia (Maiden. and Beach.) } \\
\text { Cheel., Myrtaceae, Tea tree) }\end{array}$ & Terpinen-4-ol (30.3\%) & ATCC 25922 & Gentamicin & $\mathrm{CB}(\mathrm{FICI}=0.49)$ & $S$ & \\
\hline Citronellol & - & & Clinical isolate & Penicillin & $\mathrm{CB}(\mathrm{FICI}=1.03)$ & I & {$[65]$} \\
\hline \multirow{5}{*}{ Geraniol } & \multirow{5}{*}{ - } & & Clinical isolate & Penicillin & $\mathrm{CB}(\mathrm{FICI}=1.5)$ & I & \multirow{5}{*}[60]{} \\
\hline & & & \multirow{4}{*}{ ATCC 25922} & \multirow{2}{*}{ Chloramphenicol } & $\mathrm{CB}(\mathrm{FICI}=0.32-0.87)$ & $\mathrm{S}$ & \\
\hline & & & & & $\mathrm{CB}(\mathrm{FICI}=0.98-1.09)$ & $\mathrm{Ad}$ & \\
\hline & & & & \multirow{2}{*}{ Tetracycline } & $\mathrm{CB}(\mathrm{FICI}=0.32-0.87)$ & $\mathrm{S}$ & \\
\hline & & & & & $\mathrm{CB}(\mathrm{FICI}=0.98-1.28)$ & $\mathrm{Ad}$ & \\
\hline Menthol & - & & Clinical isolate & Penicillin & $\mathrm{CB}(\mathrm{FICI}=1.5)$ & I & {$[65]$} \\
\hline Myrcene & - & & Clinical isolate & Penicillin & $\mathrm{CB}(\mathrm{FICI}=10)$ & A & \\
\hline \multirow{7}{*}{ Thymol } & & & Clinical isolate & Penicillin & $\mathrm{CB}(\mathrm{FICI}=0.15)$ & S & [65] \\
\hline & & & \multirow{6}{*}{ N00 666} & Ampicillin & $\mathrm{CB}(\mathrm{FICI}=0.12)$ & \multirow{4}{*}{ S } & \multirow{6}{*}[66]{} \\
\hline & - & & & Penicillin & $\mathrm{CB}(\mathrm{FICI}=0.20)$ & & \\
\hline & & & & Tetracycline & $\mathrm{CB}(\mathrm{FICI}=0.15)$ & & \\
\hline & & & & Erythromycin & $\mathrm{CB}(\mathrm{FICI}=0.25)$ & & \\
\hline & & & & Bacitracin & $\mathrm{CB}(\mathrm{FICI}=0.56)$ & I & \\
\hline & & & & Novobiocin & $\mathrm{CB}(\mathrm{FICI}=0.37)$ & $\mathrm{s}$ & \\
\hline
\end{tabular}


Table 2. Cont.

\begin{tabular}{|c|c|c|c|c|c|c|c|}
\hline $\begin{array}{c}\text { Essential Oil/ } \\
\text { Volatile Compound }\end{array}$ & Vegetal Source & Major Constituent & Escherichia coli Strain & Antibiotic & Method & Effect & Ref. \\
\hline \multirow{7}{*}{ Eugenol } & & \multirow{7}{*}{-} & Clinical isolate & Penicillin & $\mathrm{CB}(\mathrm{FICI}=0.16)$ & $\mathrm{S}$ & [65] \\
\hline & & & \multirow{6}{*}{ N00 666} & Ampicillin & \multirow{2}{*}{$\mathrm{CB}(\mathrm{FICI}>0.5)$} & \multirow{2}{*}{ I } & \multirow{6}{*}[66]{} \\
\hline & & & & Penicillin & & & \\
\hline & & & & Tetracycline & $\mathrm{CB}(\mathrm{FICI}=0.16)$ & $\mathrm{S}$ & \\
\hline & & & & Erythromycin & $\mathrm{CB}(\mathrm{FICI}=1.1)$ & I & \\
\hline & & & & Bacitracin & $\mathrm{CB}(\mathrm{FICI}=0.5)$ & $\mathrm{S}$ & \\
\hline & & & & Novobiocin & $\mathrm{CB}(\mathrm{FICI}=1.1)$ & I & \\
\hline \multirow{7}{*}{ Carvacrol } & & \multirow{7}{*}{-} & Clinical isolate & Penicillin & $\mathrm{CB}(\mathrm{FICI}=2)$ & I & [65] \\
\hline & & & \multirow{6}{*}{ N00 666} & Ampicillin & $\mathrm{CB}(\mathrm{FICI}=0.25)$ & \multirow{3}{*}{$\mathrm{S}$} & \multirow{6}{*}[66]{} \\
\hline & & & & Penicillin & $\mathrm{CB}(\mathrm{FICI}=0.37)$ & & \\
\hline & & & & Tetracycline & $\mathrm{CB}(\mathrm{FICI}=0.15)$ & & \\
\hline & & & & Erythromycin & $\mathrm{CB}(\mathrm{FICI}=1.0)$ & $\mathrm{I}$ & \\
\hline & & & & Bacitracin & $\mathrm{CB}(\mathrm{FICI}=0.25)$ & $\mathrm{S}$ & \\
\hline & & & & Novobiocin & $\mathrm{CB}(\mathrm{FICI}=0.63)$ & $\mathrm{I}$ & \\
\hline \multirow{6}{*}{ Cinnamaldehyde } & & \multirow{6}{*}{-} & \multirow{6}{*}{ N00 666} & Ampicillin & $\mathrm{CB}(\mathrm{FICI}=0.37)$ & \multirow{4}{*}{ S } & \multirow{12}{*}{ [66] } \\
\hline & & & & Penicillin & $\mathrm{CB}(\mathrm{FICI}=0.24)$ & & \\
\hline & & & & Tetracycline & $\mathrm{CB}(\mathrm{FICI}=0.37)$ & & \\
\hline & & & & Erythromycin & $\mathrm{CB}(\mathrm{FICI}=0.24)$ & & \\
\hline & & & & Bacitracin & $\mathrm{CB}(\mathrm{FICI}=0.63)$ & $\mathrm{I}$ & \\
\hline & & & & Novobiocin & $\mathrm{CB}(\mathrm{FICI}=0.24)$ & $\mathrm{S}$ & \\
\hline \multirow{6}{*}{ Allyl isothiocyanate } & & \multirow{6}{*}{-} & \multirow{6}{*}{ N00 666} & Ampicillin & $\mathrm{CB}(\mathrm{FICI}=0.63)$ & \multirow{4}{*}{ I } & \\
\hline & & & & Penicillin & $\mathrm{CB}(\mathrm{FICI}=1.0)$ & & \\
\hline & & & & Tetracycline & $\mathrm{CB}(\mathrm{FICI}=0.75)$ & & \\
\hline & & & & Erythromycin & $\mathrm{CB}(\mathrm{FICI}=0.73)$ & & \\
\hline & & & & Bacitracin & $\mathrm{CB}(\mathrm{FICI}=0.5)$ & S & \\
\hline & & & & Novobiocin & $\mathrm{CB}(\mathrm{FICI}=1.0)$ & I & \\
\hline
\end{tabular}

A: antagonism; Ad: addition; I: indifference; S: synergy; CB: checkerboard assay; FICI: fractional inhibitory concentration index; FR: fold reduction in MIC; MIC: minimum inhibitory concentration; IZGC (\%): percentage change in inhibition zone diameter due to gaseous contact with essential oil/volatile component. 


\section{Essential Oils and Volatile Compounds Modulating Antibiotic Activity against Klebsiella pneumoniae}

Klebsiella pneumoniae is a rod-shaped, encapsulated, non-motile, facultative anaerobic and lactose-fermenting bacillus, an opportunistic pathogen, being asymptomatically present in the gastrointestinal tract, mouth, nasopharynx and skin of healthy persons [77,78]. This Gram-negative bacterium is associated with both community-acquired and nosocomial infections, such as severe forms of pneumonia, occurring mainly in alcoholic and diabetic patients [78], pyogenic liver abscess with severe extrahepatic metastasis, including septic endophthalmitis and meningitis [79], urinary tract infections [80], nosocomial pneumonia, wound and surgical site infections, peritonitis and endocarditis, followed by subsequent bacteremia [78,81]. K. pneumoniae virulence factors include the capsule polysaccharides, fimbriae, outer membrane lipopolysaccharide and proteins and siderophores [78]. The capsule polysaccharides express antiphagocytic functions and resistance to the lytic activity of the serum complement, being essential for bacterial pathogenicity [82]. The severity of K. pneumoniae infections is also due to the biofilm formation on both biotic and abiotic surfaces $[78,82,83]$. Over the last two decades, numerous reports have described K. pneumoniae carbapenemase-producing strains. The production of these extended-spectrum $\beta$-lactamases and modification of the outer membrane permeability (porin defects, upregulation of efflux pumps) are responsible for the emergence of multidrug-resistant K. pneumoniae phenotypes [84,85]. As carbapenemases are able to hydrolyze almost all $\beta$-lactam antibiotics and even $\beta$-lactamase inhibitors, carbapenem-resistant $K$. pneumoniae remains susceptible only to a few second-line antibiotics, such as colistin, tigecycline, fosfomycin, polymyxin B and gentamicin, administered alone or in combination [83,86]. Recently, colistin-resistant K. pneumoniae has been reported in Italian hospitals [87]. In the case of infections caused by carbapenem-resistant K. pneumoniae, the combination therapy including a carbapenem and a second-line antibiotic significantly improved the patient survival $[85,88]$. Selection of second-line anti-K. pneumoniae agents should also consider particular toxicity risks for patients (e.g., colistin is known for its nephrotoxic and neurotoxic effects, and gentamicin possesses the risk of nephrotoxicity) [85].

Several EOs and volatile compounds have been reported to increase the susceptibility of K. pneumoniae reference strains or clinical isolates to conventional antibiotics (Table 3). A study investigating two endemic Moroccan thyme (Thymus maroccanus L. and Thymus broussonetii L., Lamiaceae) EOs reported synergistic effects in combination with conventional antibiotics against a K. pneumoniae clinical isolate. T. maroccanus $\mathrm{EO}$ acted synergistically with ciprofloxacin, gentamicin and pristinamycin, while T. broussonetii EO acted synergistically only with pristinamycin. The different behavior of these two EOs may be attributed to their different chemical composition: T. maroccanus EO contained a higher amount of carvacrol (76.35\%) than T. broussonetii EO (39.77\%) [57]. Thymus saturejoides Coss. (Lamiaceae) EOs, containing carvacrol as the main constituent $(25.3 \%-45.3 \%)$, acted synergistically with cefixime against a K. pneumoniae clinical isolate [76]. These synergistic effects are due to multiple targets that the combinations act on, such as the bacterial membrane (carvacrol, cefixime) [10,56], proteins (gentamicin, pristinamycin) [10], enzymes (carvacrol) [89], ATP (carvacrol) [55,56] and DNA (carvacrol, ciprofloxacin) [10,56]. Combinations between savory (Satureja kitaibelii Wierzb. ex Heuff., Lamiaceae) EO and two conventional antibiotics (chloramphenicol and tetracycline) were found to be synergistic against K. pneumoniae ATCC 700603 (10-fold reduction in the MIC values of both antibiotics). However, geraniol, the main component of savory EO (50.4\%), showed synergistic effects only in combination with chloramphenicol (10-fold reduction in the MIC value), exhibiting similar antibacterial activity to that of savory EO. As mentioned before, geraniol is a bacterial resistance modulator by targeting efflux pumps involved in antibiotic resistance $[37,60]$. Additive effects were recorded for geraniol-tetracycline combinations; it seems that other compounds in savory EO (limonene, ocimene, linalool, nerol, $\beta$-caryophyllene, germacrene D) might be involved in the synergistic interaction with tetracycline [60]. Lemon thyme (Thymus pulegioides L., Lamiaceae) $\mathrm{EO}$, belonging to geraniol/geranyl acetate chemotype, showed synergistic effects with tetracycline, streptomycin and chloramphenicol against the same reference strain, K. pneumoniae ATCC 700603 [74]. 
Miladinović et al. [90] reported synergistic effects between moon carrot (Libanotis montana Crantz, Apiaceae) EO and chloramphenicol against K. pneumoniae ATCC 700603 (10-fold reduction in the MIC value of chloramphenicol). The main components of moon carrot $\mathrm{EO}$ were sesquiterpene hydrocarbons (67.2\%), $\beta$-elemene (Figure 1) being the major constituent (40.4\%) [90]. Due to its structural analogy with cyclohexane, $\beta$-elemene disrupts the outer membrane of Gram-negative bacteria and alters the permeability of cytoplasmic and mitochondrial membranes [91]. Thus, $\beta$-elemene acts as a membrane permeabilizer and favors the chloramphenicol intrinsic mechanism of activity, namely the inhibition of protein synthesis $[10,92]$. 
Table 3. Essential oils and volatile compounds modulating the antibiotic activity against Klebsiella pneumoniae.

\begin{tabular}{|c|c|c|c|c|c|c|c|}
\hline $\begin{array}{c}\text { Essential Oil// } \\
\text { Volatile Compound }\end{array}$ & Vegetal Source & Major Constituent & Klebsiella pneumoniae Strain & Antibiotic & Method & Effect & Ref. \\
\hline \multirow{2}{*}{ Peppermint EO } & \multirow{2}{*}{$\begin{array}{l}\text { Menthae folium (Mentha piperita L., } \\
\text { Lamiaceae, Peppermint) }\end{array}$} & \multirow[t]{2}{*}{ - } & \multirow{2}{*}{ NCTC 9633} & \multirow{2}{*}{ Ciprofloxacin } & $\mathrm{CB}(\mathrm{FICI}=0.68-0.90)$ & $\mathrm{S}$ & \multirow{6}{*}[2]{} \\
\hline & & & & & $\mathrm{CB}(\mathrm{FICI}=1.40-2.24)$ & A & \\
\hline \multirow{2}{*}{ Rosemary EO } & \multirow{2}{*}{$\begin{array}{c}\text { Rosmarini folium (Rosmarinus } \\
\text { officinalis L., Lamiaceae, Rosemary) }\end{array}$} & \multirow{2}{*}{ - } & \multirow{2}{*}{ NCTC 9633} & \multirow{2}{*}{ Ciprofloxacin } & $\mathrm{CB}(\mathrm{FICI}=0.28-0.97)$ & $\mathrm{S}$ & \\
\hline & & & & & $\mathrm{CB}(\mathrm{FICI}=1.03-1.07)$ & A & \\
\hline \multirow{2}{*}{ Thyme EO } & \multirow{2}{*}{$\begin{array}{c}\text { Thymi herba (Thymus vulgaris L., } \\
\text { Lamiaceae, Thyme) }\end{array}$} & \multirow{2}{*}{ - } & \multirow{2}{*}{ NCTC 9633} & \multirow{2}{*}{ Ciprofloxacin } & $\mathrm{CB}(\mathrm{FICI}=0.71-0.90)$ & $\mathrm{S}$ & \\
\hline & & & & & $\mathrm{CB}(\mathrm{FICI}=1.10-1.40)$ & A & \\
\hline \multirow{4}{*}{ Thyme EO } & \multirow{4}{*}{$\begin{array}{l}\text { Thymi herba (Thymus maroccanus L., } \\
\text { Lamiaceae, Za'ater/Azukeni) }\end{array}$} & \multirow{4}{*}{ Carvacrol $(76.35 \%)$} & & Ciprofloxacin & $\mathrm{CB}(\mathrm{FICI}=0.37)$ & $\mathrm{S}$ & \multirow{8}{*}{ [57] } \\
\hline & & & & Gentamicin & $\mathrm{CB}(\mathrm{FICI}=0.50)$ & $\mathrm{S}$ & \\
\hline & & & & Pristinamycin & $\mathrm{CB}(\mathrm{FICI}=0.50)$ & $\mathrm{S}$ & \\
\hline & & & Clinical isolate & Cefixime & $\mathrm{CB}(\mathrm{FICI}=1)$ & I & \\
\hline \multirow{4}{*}{ Thyme EO } & \multirow{4}{*}{$\begin{array}{l}\text { Thymi herba (Thymus broussonetii L., } \\
\text { Lamiaceae, Za'atar Essaouiri) }\end{array}$} & \multirow{4}{*}{$\begin{array}{c}\text { Carvacrol }(39.77 \%) \\
\text { Borneol }(12.03 \%)\end{array}$} & & Ciprofloxacin & $\mathrm{CB}(\mathrm{FICI}=0.62)$ & PS & \\
\hline & & & & Gentamicin & $\mathrm{CB}(\mathrm{FICI}=0.62)$ & PS & \\
\hline & & & & Pristinamycin & $\mathrm{CB}(\mathrm{FICI}=0.50)$ & S & \\
\hline & & & & Cefixime & $\mathrm{CB}(\mathrm{FICI}=1)$ & I & \\
\hline \multirow{8}{*}{ Lemon thyme EO } & \multirow{8}{*}{$\begin{array}{l}\text { Thymi herba (Thymus pulegioides L., } \\
\text { Lamiaceae, Lemon } \\
\text { thyme/broad-leaved thyme) }\end{array}$} & \multirow{8}{*}{ Geraniol $(66.59 \%)$} & \multirow{8}{*}{ ATCC 700603} & \multirow{3}{*}{ Tetracycline } & $\mathrm{CB}(\mathrm{FICI}=0.76-0.82)$ & $\mathrm{S}$ & \multirow{8}{*}{ [74] } \\
\hline & & & & & $\mathrm{CB}(\mathrm{FICI}=0.92-1.10)$ & $\mathrm{Ad}$ & \\
\hline & & & & & $\mathrm{CB}(\mathrm{FICI}=1.16-1.28)$ & A & \\
\hline & & & & \multirow{3}{*}{ Streptomycin } & $\mathrm{CB}(\mathrm{FICI}=0.32-0.87)$ & $\mathrm{S}$ & \\
\hline & & & & & $\mathrm{CB}(\mathrm{FICI}=1.09)$ & $\mathrm{Ad}$ & \\
\hline & & & & & $\mathrm{CB}(\mathrm{FICI}=1.16)$ & A & \\
\hline & & & & \multirow{2}{*}{ Chloramphenicol } & $\mathrm{CB}(\mathrm{FICI}=0.32-0.87)$ & $\mathrm{S}$ & \\
\hline & & & & & $\mathrm{CB}(\mathrm{FICI}=0.98-1.09)$ & $\mathrm{Ad}$ & \\
\hline
\end{tabular}


Table 3. Cont.

\begin{tabular}{|c|c|c|c|c|c|c|c|}
\hline $\begin{array}{c}\text { Essential Oil/ } \\
\text { Volatile Compound }\end{array}$ & Vegetal Source & Major Constituent & Klebsiella pneumoniae Strain & Antibiotic & Method & Effect & Ref. \\
\hline \multirow{6}{*}{ Savory EO } & \multirow{6}{*}{$\begin{array}{l}\text { Saturejae herba (Satureja kitaibelii } \\
\text { Wierzb. ex Heuff., } \\
\text { Lamiaceae, Savory) }\end{array}$} & \multirow{6}{*}{ Geraniol (50.4\%) } & \multirow{6}{*}{ ATCC 700603} & \multirow{3}{*}{ Chloramphenicol } & $\mathrm{CB}(\mathrm{FICI}=0.21-0.43)$ & $\mathrm{S}$ & \multirow{6}{*}{60} \\
\hline & & & & & $\mathrm{CB}(\mathrm{FICI}=0.54-0.98)$ & $\mathrm{Ad}$ & \\
\hline & & & & & $\mathrm{CB}(\mathrm{FICI}=1.09)$ & $\mathrm{I}$ & \\
\hline & & & & \multirow{3}{*}{ Tetracycline } & $\mathrm{CB}(\mathrm{FICI}=0.43)$ & S & \\
\hline & & & & & $\mathrm{CB}(\mathrm{FICI}=0.56-0.98)$ & $\mathrm{Ad}$ & \\
\hline & & & & & $\mathrm{CB}(\mathrm{FICI}=1.09)$ & I & \\
\hline \multirow{3}{*}{ Thyme EO } & \multirow{3}{*}{$\begin{array}{l}\text { Thymi herba (Thymus saturejoides } \\
\text { Coss., Lamiaceae, Azoukni) }\end{array}$} & $\begin{array}{l}\text { Carvacrol }(25.3 \%) \\
\text { Borneol }(19.7 \%)\end{array}$ & \multirow{3}{*}{ Clinical isolate } & \multirow{3}{*}{ Cefixime } & $\mathrm{CB}(\mathrm{FICI}=0.75)$ & PS & \multirow{3}{*}{ [76] } \\
\hline & & $\begin{array}{l}\text { Carvacrol }(26.5 \%) \\
\text { Borneol }(20.1 \%)\end{array}$ & & & $\mathrm{CB}(\mathrm{FICI}=0.75)$ & PS & \\
\hline & & $\begin{array}{l}\text { Carvacrol }(45.3 \%) \\
\text { Borneol }(7.5 \%)\end{array}$ & & & $\mathrm{CB}(\mathrm{FICI}=0.50)$ & S & \\
\hline Sandarac EO & $\begin{array}{c}\text { Tetraclinis coni (Tetraclinis articulata } \\
\text { (Vahl.) Masters., } \\
\text { Cupressaceae, Sandarac) }\end{array}$ & $\begin{array}{c}\alpha \text {-Campholenal }(16.34 \%) \\
\text { Trans-Pinocarveol }(15.45 \%) \\
\text { Verbenone }(13.36 \%) \\
\text { Cis-Verbenol }(12.36 \%)\end{array}$ & CIP 8291 & Amoxicillin & $\mathrm{CB}(\mathrm{FICI}=0.8)$ & PS & [75] \\
\hline \multirow{2}{*}{ Tea tree EO } & \multirow{2}{*}{$\begin{array}{c}\text { Melaleucae folium (Melaleuca } \\
\text { alternifolia, (Maiden. and Betch.) } \\
\text { Chee., Myrtaceae, Tea tree) }\end{array}$} & \multirow{2}{*}{ - } & \multirow{2}{*}{ NCTC 9633} & \multirow{2}{*}{ Ciprofloxacin } & $\mathrm{CB}(\mathrm{FICI}=0.73-0.95)$ & $\mathrm{S}$ & \multirow{2}{*}{ [2] } \\
\hline & & & & & $\mathrm{CB}(\mathrm{FICI}=1.03-1.85)$ & A & \\
\hline \multirow{5}{*}{ Geraniol } & \multirow[t]{5}{*}{ 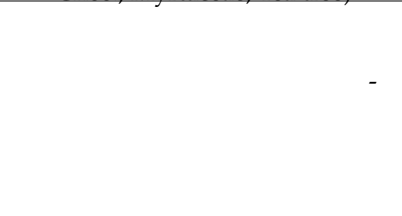 } & & \multirow{5}{*}{ ATCC 700603} & \multirow[t]{2}{*}{ Chloramphenicol } & $\mathrm{CB}(\mathrm{FICI}=0.32-0.87)$ & $\mathrm{S}$ & \multirow{5}{*}{ [60] } \\
\hline & & & & & $\mathrm{CB}(\mathrm{FICI}=0.98-1.09)$ & $\mathrm{Ad}$ & \\
\hline & & & & \multirow{3}{*}{ Tetracycline } & $\mathrm{CB}(\mathrm{FICI}=0.76-0.80)$ & $S$ & \\
\hline & & & & & $\mathrm{CB}(\mathrm{FICI}=0.95-1.10)$ & $\mathrm{Ad}$ & \\
\hline & & & & & $\mathrm{CB}(\mathrm{FICI}=1.25-1.66)$ & A & \\
\hline
\end{tabular}

A: antagonism; Ad: addition; I: indifference; PS: partial synergy; S: synergy; CB: checkerboard assay; FICI: fractional inhibitory concentration index. 


\section{Essential Oils and Volatile Compounds Modulating Antibiotic Activity against Pseudomonas aeruginosa}

Pseudomonas aeruginosa is an aerobic, rod-shaped, non-spore forming, oxidase positive and non-lactose fermenting Gram-negative bacterium [93]. It is a common agent for nosocomial infections being responsible for urinary tract, corneal, surgical and wound infections, but also for pneumonia and endocarditis. This pathogen is involved in chronic respiratory infections in cystic fibrosis patients. In addition, it is the second pathogen responsible for ventilator-associated pneumonia [94]. Bacteremia caused by P. aeruginosa leads to a mortality rate of $30 \%-50 \%$ in cancer patients who develop neutropenia after chemotherapy $[95,96]$. The pili, proteases, exoenzyme $S$, siderophores and alginate pseudocapsule are the main virulence factors of P. aeruginosa [97]. Amoxicillin, first and second generation cephalosporins are naturally inactive against $P$. aeruginosa. The most problematic aspect is the resistance of $P$. aeruginosa strains to many antibiotics. Piperacillin and ceftazidime resistance is explained by $\beta$-lactamase production, but strains resistant to quinolones and aminoglycosides have also been reported [95]. Besides the alteration of antibiotic target, another mechanism responsible for antibiotic resistance is the efflux pump overexpression [98].

Numerous studies have been conducted in order to assess if combinations between EOs and conventional antibiotics are able to increase the antibiotic susceptibility of $P$. aeruginosa strains (Table 4). Strong synergistic effects have been reported for the combinations between basil (Ocimum basilicum L., Lamiaceae) EO and imipenem/ciprofloxacin against P. aeruginosa ATCC 25853 and the 1662339 clinical isolate. Both linalool, the main ingredient of basil EO (55.2\%), and imipenem act on the same target, the bacterial cell membrane, whereas ciprofloxacin inhibits DNA gyrase and topoisomerase IV $[99,100]$. Thyme (Thymus vulgaris L., Lamiaceae) and marjoram (Origanum majorana L., Lamiaceae) EOs restored piperacillin susceptibility of P. aeruginosa ATCC 9027 (resistant to piperacillin, but susceptible to cefepime, meropenem, gentamicin and norfloxacin). Both EOs acted synergistically in combination with cefepime, meropenem and gentamicin. Thyme and marjoram EOs had high contents of oxygenated terpenes, thymol (33.6\%) and 4-terpineol (21.3\%), respectively. Weaker synergistic effects have been detected for sage (Salvia officinalis L., Lamiaceae) EO in combination with piperacillin, meropenem and gentamicin. 1,8-Cineole (29\%) (Figure 1) was the major oxygenated terpene in sage EO [101]. Alecrim pimento (Lippia sidoides Cham., Verbenaceae) $\mathrm{EO}$, rich in thymol $(84.9 \%)$, reduced the MIC values of gentamicin and neomycin by four- and two-fold, respectively [102]. Phenolic terpenoids, such as thymol, alter the bacterial membrane, facilitating the antibiotic uptake. Moreover, thymol is distributed in the bilayer lipid membrane causing loss of $\mathrm{K}^{+}$and ATP [103]. Additional mechanisms involve the alteration of periplasmic proteins and the citrate metabolic pathway [104]. Monoterpene alcohols, such as 4-terpineol, also have antipseudomonal activity, as they inhibit the microbial oxygen uptake and oxidative phosphorylation. Terpenoid ethers (1,8-cineole) have a lower capacity to sensitize the pathogen to the antibiotic activity as they lack free hydroxyl groups, a structural feature present in both phenolic and alcoholic terpenoids [101,105]. Three Thymus saturejoides Coss. (Lamiaceae) EOs significantly increased the susceptibility of AmpC $\beta$-lactamase producing P. aeruginosa ATCC 27853 to cefixime. All EOs were characterized by high contents of carvacrol $(25.3 \%, 26.5 \%$ and $45.3 \%)$ and borneol $(19.7 \%, 20.1 \%$ and 7.5\%) [76]. Other carvacrol-rich EOs, Thymus maroccanus L. and Thymus broussonetii L. (Lamiaceae) EOs, acted synergistically with ciprofloxacin and gentamicin against a P. aeruginosa clinical isolate. These synergistic effects are mainly due to carvacrol, a cytoplasmic membrane permeabilizer and an ATP, DNA and enzyme inhibitor [55-57]. Lippia gracilis Schauer. (Verbenaceae) EO, containing thymol $(44.4 \%)$ and carvacrol $(22.2 \%)$ as major constituents, significantly increased the susceptibility of P. aeruginosa ATCC 15442 to amikacin, tobramycin and gentamicin if the combinations were tested by direct contact. A marked difference was detected when tested in gaseous contact: high dilutions of Lippia gracilis $\mathrm{EO}(25 \%, 12 \%$ and $6 \%)$ increased the activity of gentamycin, but did not increase the activity of tobramycin. Tobramycin and gentamicin belong to the same class of antibiotics, but tobramycin is more polar than gentamicin, and it can hardly cross the lipid membrane. Moreover, 
in contrast with direct contact, in the case of gaseous contact, the interaction between bacteria and EO vapors is significantly influenced by the concentration of the latter [106]. It is possible that the high dilutions of Lippia gracilis EO used in the study were not enough to facilitate the entrance of tobramycin within the cell [107]. The susceptibility of P. aeruginosa ATCC 15442 to tobramycin, amikacin and gentamicin was increased by gaseous contact with Hyptis martiusii Benth. (Verbenaceae) EO. The synergistic effect with amikacin in gaseous contact was converted into an antagonistic one in the case of direct contact. A possible explanation would be related to the fact that the gaseous contact involves a series of qualitative and quantitative changes in EO composition. Unstable volatile constituents undergo degradation, generating new substances with different biological effects; for example, bicyclogermacrene converts into spathulenol, which increases the antibacterial activity of Hyptis martiusii EO [64]. Synergistic effects against the same $\beta$-lactamase producing strain (P. aeruginosa ATCC 27853) have been reported for geraniol, savory (Satureja kitaibelii Wierzb. ex Heuff., Lamiaceae) and lemon thyme (Thymus pulegioides L., Lamiaceae) EOs in combination with chloramphenicol or tetracycline. Geraniol is a major constituent in savory and lemon thyme EOs (50.4\% and 66.59\%, respectively) [60,74]. Immortelle (Helichrysum italicum (Roth) G. Don., Asteraceae) EO restored the susceptibility to chloramphenicol of two highly chloramphenicol-resistant P. aeruginosa strains (PAO1 and PA124). Immortelle EO decreased the MICs of chloramphenicol against PAO1 and PA124 by $16-$ and eight-fold, respectively. These effects are undoubtedly due to the ability of geraniol, a constituent of EO, to inhibit efflux pumps responsible for drug resistance. As a result, geraniol increased the intra-bacterial concentration of antibiotics [37]. Nevertheless, lemon thyme EO acted antagonistically with streptomycin. Antagonism is often due to a competition for the same target. It is possible that some constituents in lemon thyme EO have the same target as streptomycin, namely the 30S ribosomal subunit [74]. P. aeruginosa ATCC 15442 is usually used to assess the effectiveness of disinfectants and antimicrobial preservatives. Gentamicin activity against this bacterial strain was significantly enhanced by gaseous contact with oxygenated sesquiterpene-rich EOs (Zanthoxylum articulatum, Vanillosmopsis arborea, Croton zehntneri EOs) [106,108,109]. Oxygenated sesquiterpenes (viridiflorol, $\alpha$-bisabolol, trans-anethole) (Figure 1) are lipophilic compounds that accumulate into the phospholipid bilayers of bacterial membrane, causing its disruption, with a subsequent increase in the penetration of hydrophilic substances, such as gentamicin [110,111]. Viridiflorol exerts antibacterial activity through an additional mechanism, including interference with the respiratory chain reaction and energy production [106]. Antagonistic effects have been detected for Vanillosmopsis arborea EO in combination with tetracycline or tobramycin (gaseous contact). These effects could be attributed to chemical interactions between EO constituents and tetracycline/tobramycin, which led to antibiotic inactivation [108]. Similar results have been reported for Lippia microphylla EO, rich in 1,8-cineole (18.1\%) and $\beta$-ocimene (15.2\%) [112]. A strong synergistic effect against P. aeruginosa ATCC 15442 was recorded for amikacin in association with Lantana montevidensis Briq. (Verbenaceae) EO containing $31.5 \% \beta$-caryophyllene (Figure 1). This non-polar compound might also affect the bacterial membrane and interfere with the mitochondrial respiratory chain reaction $[113,114]$. It is worthy to note eugenol (Figure 1), a volatile phenylpropanoid, which enhanced the activity of different penicillins, fluoroquinolones and macrolides against P. aeruginosa NCIM 5029. Bacterial cells treated with eugenol at a concentration of $1 \mathrm{mM}$ had a 50\% loss of membrane integrity. Therefore, eugenol synergized with penicillins, antibiotics targeting the bacterial wall. Acting as a membrane permeabilizer, eugenol facilitated the uptake of antibiotics that target ribosome (erythromycin, tetracycline, chloramphenicol, rifampicin) or DNA synthesis (norfloxacin). The synergistic effect could also be attributed to the intrinsic antimicrobial activity of eugenol. The hydroxyl group of eugenol is responsible for the inhibition of some enzymes, such as ATP-ase, histidine decarboxylase or proteases, thus impairing the bacterial metabolism [104,115]. 
Table 4. Essential oils and volatile compounds modulating the antibiotic activity against Pseudomonas aeruginosa.

\begin{tabular}{|c|c|c|c|c|c|c|c|}
\hline $\begin{array}{c}\text { Essential Oil// } \\
\text { Volatile Compound }\end{array}$ & Vegetal Source & Major Constituent & Pseudomonas aeruginosa Strain & Antibiotic & Method & Effect & Ref. \\
\hline \multirow{4}{*}{ Thyme EO } & \multirow{4}{*}{$\begin{array}{l}\text { Thymi herba (Thymus maroccanus L., } \\
\text { Lamiaceae, Za'ater/Azukeni) }\end{array}$} & \multirow{4}{*}{ Carvacrol $(76.35 \%)$} & \multirow{8}{*}{ Clinical isolate } & Ciprofloxacin & $\mathrm{CB}(\mathrm{FICI}=0.15)$ & $\mathrm{s}$ & \multirow{8}{*}[57]{} \\
\hline & & & & Gentamicin & $\mathrm{CB}(\mathrm{FICI}=0.18)$ & $\mathrm{S}$ & \\
\hline & & & & Pristinamycin & $\mathrm{CB}(\mathrm{FICI}=0.75)$ & PS & \\
\hline & & & & Cefixime & $\mathrm{CB}(\mathrm{FICI}=0.75)$ & PS & \\
\hline \multirow{4}{*}{ Za'atar Essaouiri EO } & \multirow{4}{*}{$\begin{array}{l}\text { Thymi herba (Thymus broussonetii L., } \\
\text { Lamiaceae, Za'atar Essaouiri) }\end{array}$} & \multirow{4}{*}{$\begin{array}{l}\text { Carvacrol }(39.77 \%) \\
\text { Borneol }(12.03 \%)\end{array}$} & & Ciprofloxacin & $\mathrm{CB}(\mathrm{FICI}=0.14)$ & $\mathrm{S}$ & \\
\hline & & & & Gentamicin & $\mathrm{CB}(\mathrm{FICI}=0.28)$ & $\mathrm{S}$ & \\
\hline & & & & Pristinamycin & $\mathrm{CB}(\mathrm{FICI}=0.75)$ & PS & \\
\hline & & & & Cefixime & $\mathrm{CB}(\mathrm{FICI}=0.5)$ & $\mathrm{s}$ & \\
\hline \multirow{6}{*}{ Lemon thyme EO } & \multirow{6}{*}{$\begin{array}{l}\text { Thymi herba (Thymus pulegioides L., } \\
\text { Lamiaceae, Lemon thyme/ } \\
\text { broad-leaved thyme) }\end{array}$} & \multirow{6}{*}{ Geraniol (66.59\%) } & \multirow{6}{*}{ ATCC 27853} & \multirow{3}{*}{ Tetracycline } & $\mathrm{CB}(\mathrm{FICI}=0.54-0.82)$ & $\mathrm{S}$ & \multirow{6}{*}[74]{} \\
\hline & & & & & $\mathrm{CB}(\mathrm{FICI}=0.95-1.08)$ & $\mathrm{Ad}$ & \\
\hline & & & & & $\mathrm{CB}(\mathrm{FICI}=1.16-1.28)$ & $\mathrm{A}$ & \\
\hline & & & & Streptomycin & $\mathrm{CB}(\mathrm{FICI}=1.20-2.00)$ & A & \\
\hline & & & & \multirow{2}{*}{ Chloramphenicol } & $\mathrm{CB}(\mathrm{FICI}=0.43-0.87)$ & $\mathrm{S}$ & \\
\hline & & & & & CB (FICI $=0.98-1.09)$ & $\mathrm{Ad}$ & \\
\hline \multirow{4}{*}{ Savory EO } & \multirow{4}{*}{$\begin{array}{l}\text { Saturejae herba (Satureja kitaibelii } \\
\text { Wierzb. ex Heuff., Lamiaceae, Savory) }\end{array}$} & \multirow{4}{*}{ Geraniol (50.4\%) } & \multirow{4}{*}{ ATCC 27853} & \multirow{3}{*}{ Tetracycline } & $\mathrm{CB}(\mathrm{FICI}=0.54-0.82)$ & $\mathrm{S}$ & \multirow{4}{*}[60]{} \\
\hline & & & & & $\mathrm{CB}(\mathrm{FICI}=0.96-1.10)$ & $\mathrm{Ad}$ & \\
\hline & & & & & $\mathrm{CB}(\mathrm{FICI}=1.21-1.47)$ & A & \\
\hline & & & & Chloramphenicol & $\mathrm{CB}(\mathrm{FICI}=0.98-1.09)$ & Ad & \\
\hline \multirow{3}{*}{ Thyme EO } & \multirow{3}{*}{$\begin{array}{c}\text { Thymi herba (Thymus saturejoides Coss., } \\
\text { Lamiaceae, Azoukni) }\end{array}$} & $\begin{array}{l}\text { Carvacrol }(25.3 \%) \\
\text { Borneol }(19.7 \%)\end{array}$ & \multirow{3}{*}{ ATCC 27853} & \multirow{3}{*}{ Cefixime } & $\mathrm{CB}(\mathrm{FICI}=0.28)$ & \multirow{3}{*}{ S } & \multirow{3}{*}[76]{} \\
\hline & & $\begin{array}{l}\text { Carvacrol }(26.5 \%) \\
\text { Borneol }(20.1 \%)\end{array}$ & & & $\mathrm{CB}(\mathrm{FICI}=0.31)$ & & \\
\hline & & $\begin{array}{c}\text { Carvacrol }(45.3 \%) \\
\text { Borneol }(7.5 \%)\end{array}$ & & & $\mathrm{CB}(\mathrm{FICI}=0.29)$ & & \\
\hline Sandarac EO & $\begin{array}{l}\text { Tetraclinis coni (Tetraclinis articulata (Vahl.) } \\
\text { Masters., Cupressaceae, Sandarac) }\end{array}$ & $\begin{array}{c}\alpha \text {-Campholenal }(16.34 \%) \\
\text { Trans-Pinocarveol }(15.45 \%) \\
\text { Verbenone }(13.36 \%) \\
\text { Cis-Verbenol }(12.36 \%)\end{array}$ & CIPA 22 & Amoxicillin & $\mathrm{CB}(\mathrm{FICI}=1.00)$ & $\mathrm{Ad}$ & [75] \\
\hline
\end{tabular}


Table 4. Cont.

\begin{tabular}{|c|c|c|c|c|c|c|c|}
\hline $\begin{array}{c}\text { Essential Oil/ } \\
\text { Volatile Compound }\end{array}$ & Vegetal Source & Major Constituent & Pseudomonas aeruginosa Strain & Antibiotic & Method & Effect & Ref. \\
\hline \multirow{7}{*}{$\begin{array}{l}\text { Cidreira-do-mato/ } \\
\text { cidreira-brava EO }\end{array}$} & \multirow{7}{*}{$\begin{array}{l}\text { Hyptis folium (Hyptis martiusii Benth., } \\
\text { Lamiaceae, } \\
\text { Cidreira-do-mato/cidreira-brava) }\end{array}$} & \multirow{7}{*}{$\begin{array}{c}\text { Bicyclogermacrene }(10.6 \%) \\
\text { Trans-Caryophyllene }(9.2 \%) \\
\text { Caryophyllene oxide }(7.4 \%) \\
\text { 1,8-Cineole }(7 \%)\end{array}$} & \multirow{7}{*}{ ATCC 15442} & \multirow{2}{*}{ Gentamycin } & IZGC (+18.9\%) & $\mathrm{S}$ & \multirow{7}{*}{ [64] } \\
\hline & & & & & FR $(-)$ & I & \\
\hline & & & & Amikacin & IZGC $(+60 \%)$ & S & \\
\hline & & & & Amikacin & FR4-fold increase in MIC & A & \\
\hline & & & & Tobramycin & IZGC (+12.5\%) & $\mathrm{s}$ & \\
\hline & & & & Neomycin & FR (-) & I & \\
\hline & & & & Kanamycin & FR (-) & I & \\
\hline \multirow{4}{*}{ Marjoram EO } & \multirow{4}{*}{$\begin{array}{l}\text { Origani folium (Origanum majorana L., } \\
\text { Lamiaceae, Marjoram) }\end{array}$} & \multirow{4}{*}{ 4-Terpineol $(21.3 \%)$} & \multirow{12}{*}{ ATCC 9027} & Cefepime & IZDC $(+10 \%)$ & $\mathrm{S}$ & \multirow{12}{*}{ [101] } \\
\hline & & & & Meropenem & IZDC (+20.5\%) & $\mathrm{s}$ & \\
\hline & & & & Gentamicin & $\operatorname{IZDC}(+31.03 \%)$ & $\mathrm{s}$ & \\
\hline & & & & Norfloxacin & $\operatorname{IZDC}(-0.03 \%)$ & A & \\
\hline \multirow{5}{*}{ Sage EO } & \multirow{5}{*}{$\begin{array}{l}\text { Salviae folium (Salvia officinalis L., } \\
\text { Lamiaceae, Sage) }\end{array}$} & \multirow{5}{*}{ 1,8-Cineole (29\%) } & & Piperacillin & IZDC (+29.4\%) & $\mathrm{s}$ & \\
\hline & & & & Cefepime & $\operatorname{IZDC}(-0.03 \%)$ & A & \\
\hline & & & & Meropenem & $\operatorname{IZDC}(+23.5 \%)$ & $\mathrm{s}$ & \\
\hline & & & & Gentamicin & $\operatorname{IZDC}(+13.7 \%)$ & $\mathrm{s}$ & \\
\hline & & & & Norfloxacin & $\operatorname{IZDC}(-0.16 \%)$ & A & \\
\hline \multirow[t]{3}{*}{ Thyme EO } & \multirow{3}{*}{$\begin{array}{l}\text { Thymi herba (Thymus vulgaris L., } \\
\text { Lamiaceae, Thyme) }\end{array}$} & \multirow[t]{3}{*}{ Thymol (33.6\%) } & & Cefepime & IZDC $(+53.3 \%)$ & $\mathrm{S}$ & \\
\hline & & & & Meropenem & IZDC $(+52.9 \%)$ & $\mathrm{s}$ & \\
\hline & & & & Gentamicin & IZDC (+37.9\%) & $\mathrm{s}$ & \\
\hline \multirow{4}{*}{ Basil EO } & \multirow{4}{*}{$\begin{array}{c}\text { Basilici herba (Ocimum basilicum L., } \\
\text { Lamiaceae, Basil) }\end{array}$} & \multirow{4}{*}{ Linalool (55.2\%) } & \multirow{2}{*}{ ATCC 25853} & Imipenem & $\mathrm{CB}(\mathrm{FICI}=0.75)$ & $\mathrm{Ad}$ & \multirow{4}{*}{ [99] } \\
\hline & & & & Ciprofloxacin & $\mathrm{CB}(\mathrm{FICI}=1.03)$ & I & \\
\hline & & & \multirow{2}{*}{1662339} & Imipenem & $\mathrm{CB}(\mathrm{FICI}=0.0625)$ & $\mathrm{s}$ & \\
\hline & & & & Ciprofloxacin & $\mathrm{CB}(\mathrm{FICI}=0.09)$ & $\mathrm{s}$ & \\
\hline \multirow{2}{*}{ Alecrim-de-tabuleiro EO } & \multirow{2}{*}{$\begin{array}{l}\text { Lippiae folium (Lippia microphylla Cham., } \\
\text { Verbenaceae, Alecrim-de-tabuleiro) }\end{array}$} & \multirow{2}{*}{$\begin{array}{c}\text { 1,8-Cineole }(18.1 \%) \\
\text { Z- } \beta \text {-Ocimene }(15.2 \%) \\
\text { Bicyclogermacrene }(11.6 \%)\end{array}$} & \multirow{2}{*}{ ATCC 15442} & Gentamicin & IZGC $(+47 \%)$ & $\mathrm{S}$ & \multirow[t]{2}{*}{ [112] } \\
\hline & & & & Tetracycline & $\operatorname{IZGC}(-14 \%)$ & A & \\
\hline \multirow{2}{*}{ Creeping lantana EO } & Lantanae folium (Lantana montevidensis Briq., & $\beta$-Caryophyllene $(31.50 \%)$ & ATCC 15442 & Gentamicin & IZGC $(+12 \%)$ & $\mathrm{s}$ & [113] \\
\hline & & Germacrene D (27.50\%) & & Amikacin & $\operatorname{IZGC}(+102 \%)$ & & \\
\hline Alecrim pimento EO & Lippiae folium (Lippia sidoides Cham., & Thymol (84.9\%) & ATCC 15442 & Gentamycin & FR (4-fold reduction in MIC) & $\mathrm{s}$ & [102] \\
\hline & Verbenaceae, Alecrim pimento) & & & Neomycin & FR (2-fold reduction in MIC) & & \\
\hline
\end{tabular}


Table 4. Cont.

\begin{tabular}{|c|c|c|c|c|c|c|c|}
\hline $\begin{array}{c}\text { Essential Oil/ } \\
\text { Volatile Compound }\end{array}$ & Vegetal Source & Major Constituent & Pseudomonas aeruginosa Strain & Antibiotic & Method & Effect & Ref. \\
\hline \multirow{3}{*}{ Alecrim-da-chapada EO } & \multirow{3}{*}{$\begin{array}{l}\text { Lippiae folium (Lippia gracilis Schauer., } \\
\text { Verbenaceae, Alecrim-da-chapada) }\end{array}$} & \multirow{3}{*}{$\begin{array}{l}\text { Thymol }(44.4 \%) \\
\text { Carvacrol }(22.2 \%)\end{array}$} & \multirow{3}{*}{ ATCC 15442} & Amikacin & IZGC & \multirow{3}{*}{$\mathrm{s}$} & \multirow{3}{*}{ [107] } \\
\hline & & & & Tobramycin & IZGC & & \\
\hline & & & & Gentamycin & IZGC & & \\
\hline \multirow{3}{*}{ Candeeiro EO } & \multirow{3}{*}{$\begin{array}{l}\text { Vanillosmopsis cortex (Vanillosmopsis arborea } \\
\text { Baker., Asteraceae Candeeiro) }\end{array}$} & \multirow{3}{*}{$\alpha$-Bisabolol $(80.43 \%)$} & \multirow{3}{*}{ ATCC 15442} & Gentamycin & $\operatorname{IZGC}(+8.6 \%)$ & $\mathrm{s}$ & \multirow{3}{*}{ [108] } \\
\hline & & & & Tetracycline & $\operatorname{IZGC}(-8.0 \%)$ & A & \\
\hline & & & & Tobramycin & IZGC $(-18.0 \%)$ & A & \\
\hline Immortelle EO & $\begin{array}{l}\text { Helichrysi flos (Helichrysum italicum (Roth) G. } \\
\text { Don, Asteraceae, Immortelle) }\end{array}$ & - & PAO1 PA124 & Chloramphenicol & FR (16-fold reduction in MIC) & $\mathrm{S}$ & {$[37]$} \\
\hline \multirow{2}{*}{ Canela de Cunha EO } & \multirow{2}{*}{$\begin{array}{l}\text { Crotonii folium (Croton zehntneri Pax et } \\
\text { Hoffm., Euphorbiaceae Canela de Cunha) }\end{array}$} & \multirow{2}{*}{ Trans-Anethole } & \multirow{2}{*}{ ATCC 15442} & Gentamycin & IZGC $(+42.8 \%)$ & $\mathrm{S}$ & \multirow{2}{*}{ [109] } \\
\hline & & & & Tetracycline & IZGC $(0 \%)$ & I & \\
\hline \multirow{2}{*}{ Limão-bravo EO } & \multirow{2}{*}{$\begin{array}{l}\text { Zanthoxylii folium (Zanthoxylum articulatum } \\
\text { Engler Rutaceae, Limão-bravo) }\end{array}$} & \multirow{2}{*}{ Viridiflorol (35.4\%) } & \multirow{2}{*}{ ATCC 15442} & Gentamycin & IZGC $(+43.8 \%)$ & $\mathrm{s}$ & \multirow{2}{*}{ [106] } \\
\hline & & & & Tetracycline & $\operatorname{IZGC}(+9.6 \%)$ & $\mathrm{Ad}$ & \\
\hline \multirow{7}{*}{ Eugenol } & \multirow{7}{*}{ - } & & \multirow{7}{*}{ NCIM 5029} & Ampicillin & \multirow{7}{*}{$\mathrm{CB}(\mathrm{FICI}<0.50)$} & & \\
\hline & & & & Chloramphenicol & & & \\
\hline & & & & Erythromycin & & & \\
\hline & & & & Norfloxacin & & $\mathrm{s}$ & [115] \\
\hline & & & & Polymyxin & & & \\
\hline & & & & Rifampicin & & & \\
\hline & & & & Erythromycin & & & \\
\hline Thymol & - & & ATCC 15442 & Gentamycin & FR (4-fold reduction in MIC) & $S$ & {$[102]$} \\
\hline & & & & Neomycin & FR (2-fold reduction in MIC) & & \\
\hline & & & & Chloramphenicol & $\mathrm{CB}(\mathrm{FICI}=0.54-0.87)$ & $\mathrm{s}$ & \\
\hline Geraniol & - & & ATCC 27853 & & $\mathrm{CB}(\mathrm{FICI}=0.92-1.09)$ & $\mathrm{Ad}$ & {$[60]$} \\
\hline (5) & & & & & CB $(\mathrm{FICI}=0.76)$ & $\mathrm{s}$ & \\
\hline & & & & Tetracycline & $\mathrm{CB}(\mathrm{FICI}=0.92-1.10)$ & $\mathrm{Ad}$ & \\
\hline & & & & & $\mathrm{CB}(\mathrm{FICI}=1.16-1.47)$ & A & \\
\hline
\end{tabular}

A: antagonism; Ad: addition; I: indifference; PS: partial synergy; S: synergy; CB: checkerboard assay; FICI: fractional inhibitory concentration index; FR: fold reduction in MIC; MIC minimum inhibitory concentration; IZGC (\%): percentage change in inhibition zone diameter due to gaseous contact with essential oil/volatile component; IZDC (\%): percentage change in inhibition zone diameter due to direct contact with essential oil/volatile component. 


\section{Essential Oils and Volatile Compounds Modulating Antibiotic Activity against Other Gram-Negative Bacteria}

Enterobacter aerogenes and E. cloacae are facultative anaerobic, rod-shaped and non-spore-forming Gram-negative bacteria with clinical significance, being responsible for hospital-acquired infections [116,117]. These infections are usually associated with a high fatality rate due to antibiotic resistance. E. aerogenes and E. cloacae are naturally resistant to aminopenicillins, and numerous clinical isolates exhibited resistance to other $\beta$-lactam antibiotics due to the production of extended-spectrum $\beta$-lactamases. Analysis of several E. aerogenes clinical isolates revealed resistance to quinolones, tetracycline and chloramphenicol due to the overexpression of efflux mechanisms [118]. Several EOs have been evaluated in order to assess their potential as antibiotic resistance modifying agents for $E$. aerogenes and E. cloacae strains (Table 5). Thymus maroccanus L. and Thymus broussonetii L. (Lamiaceae) EOs decreased the MIC value of chloramphenicol against E. aerogenes ATCC 13048 and the EA27 clinical isolate (overexpressing AcrAB pump responsible for multidrug-resistance) by four- and eight-fold, respectively. The results were comparable to those obtained with $\mathrm{Pa} \beta \mathrm{N}$. It seems that both EOs inhibited the efflux pumps [52]. For T. maroccanus EO, there has also been reported a membranotropic effect on Gram-negative bacteria involving the permeabilization of both inner and outer bacterial membranes, whereas polymyxin B permeabilizes only the outer membrane; no degradation of cellular constituents was detected [119]. Both thyme EOs acted synergistically with ciprofloxacin, gentamicin and pristinamycin against an E. cloacae clinical isolate [57]. Carvacrol, the main component of both EOs $(76.35 \%$ and $39.77 \%$, respectively), is known to increase the bacterial membrane permeability and inhibit ATP-ase activity [10], thus facilitating the activity of conventional antibiotics that act on different targets. Immortelle (Helichrysum italicum (Roth) G. Don., Asteraceae) EO increased the susceptibility to chloramphenicol of several E. aerogenes strains: E. aerogenes ATCC 13048 (susceptible to many antibiotics, but resistant to amoxicillin), E. aerogenes EAEP289 (overexpressing the AcrAB pump, but susceptible to kanamycin), E. aerogenes EAEP294 (having the AcrAB pump deleted, but expressing other efflux pumps involved in antibiotic resistance) and E. aerogenes CM-64 (overexpressing the AcrAB pump). It is worthy to note a significant reduction in the MIC of chloramphenicol against the E. aerogenes EAEP294 strain (128-fold reduction). Geraniol, a component of immortelle EO, has been reported to increase the susceptibility of E. aerogenes EAEP294 to other antibiotics (ampicillin, penicillin, norfloxacin) [37]. Obviously, the efflux pump inhibitory activity of geraniol plays an important role in the synergy between immortelle EO and antibiotics against E. aerogenes strains. Synergistic effects against E. aerogenes NCIM 5139 have been reported for eugenol in combination with antibiotics acting on different targets $[115,120]$.

Proteus vulgaris is an aerobic and facultatively anaerobic member of the Enterobacteriaceae family. Urinary tract, burn, bloodstream, wound and respiratory tract infections are the most common infections caused by P. vulgaris. Adherence through pili or fimbriae and cytotoxic hemolysins are the most important pathogenicity factors of $P$. vulgaris [121,122]. P. vulgaris is naturally resistant to benzylpenicillin, oxacillin, macrolides and tetracycline. Acquired resistance has been reported for ampicillin, third-generation cephalosporins (ceftazidime, cefotaxime, ceftriaxone), but also for aztreonam, monobactam due to extended-spectrum $\beta$-lactamase production. P. vulgaris strains are usually susceptible to aminoglycosides and quinolones [123]. Eugenol has been reported to act synergistically in combination with several antibiotics against $P$. vulgaris NCIM 2813 (Table 5). 
Table 5. Essential oils and volatile compounds modulating the antibiotic activity against other Gram-negative bacteria.

\begin{tabular}{|c|c|c|c|c|c|c|c|}
\hline $\begin{array}{l}\text { Essential } \\
\text { Oil/Volatile } \\
\text { Compound }\end{array}$ & Vegetal Source & Major Constituent & $\begin{array}{c}\text { Other Gram-negative } \\
\text { Bacteria }\end{array}$ & Antibiotic & Method & Effect & Ref. \\
\hline \multicolumn{8}{|c|}{$\begin{array}{c}\text { Enterobacter aerogenes } \\
\text { Strain }\end{array}$} \\
\hline \multirow{2}{*}{ Thyme EO } & \multirow{2}{*}{$\begin{array}{l}\text { Thymi herba (Thymus maroccanus L., } \\
\text { Lamiaceae, Za'ater/Azukeni) }\end{array}$} & \multirow{2}{*}{ Carvacrol $(76.35 \%)$} & ATCC 13048 & \multirow{4}{*}{ Chloramphenicol } & FR (4-fold reduction in MIC) & \multirow{4}{*}{ S } & \multirow{4}{*}{ [52] } \\
\hline & & & EA27clinical isolate & & FR (8-fold reduction in MIC) & & \\
\hline \multirow{2}{*}{ Thyme EO } & \multirow{2}{*}{$\begin{array}{l}\text { Thymi herba (Thymus broussonetii L., } \\
\text { Lamiaceae, Za'atar Essaouiri) }\end{array}$} & \multirow{2}{*}{$\begin{array}{l}\text { Carvacrol }(39.77 \%) \\
\text { Borneol }(12.03 \%)\end{array}$} & ATCC 13048 & & FR (4-fold reduction in MIC) & & \\
\hline & & & EA27clinical isolate & & FR (8-fold reduction in MIC) & & \\
\hline \multirow{9}{*}{ Immortelle EO } & \multirow{9}{*}{$\begin{array}{l}\text { Helichrysi flos (Helichrysum italicum } \\
\text { (Roth) G. Don, Asteraceae, } \\
\text { Immortelle) }\end{array}$} & \multirow{9}{*}{-} & ATCC 13048 & Chloramphenicol & FR (2-fold reduction in MIC) & \multirow{2}{*}{ S } & \multirow{9}{*}{ [37] } \\
\hline & & & \multirow{4}{*}{ EAEP289 } & Chloramphenicol & FR (8-fold reduction in MIC) & & \\
\hline & & & & Ampicillin & FR $(-)$ & \multirow{2}{*}{ I } & \\
\hline & & & & Penicillin & FR $(-)$ & & \\
\hline & & & & Norfloxacin & FR (2-fold reduction in MIC) & \multirow{5}{*}{ S } & \\
\hline & & & \multirow{4}{*}{ EAEP294 } & Chloramphenicol & FR (128-fold reduction in MIC) & & \\
\hline & & & & Ampicillin & $\begin{array}{l}\text { FR ( 7300-fold reduction in } \\
\text { MIC) }\end{array}$ & & \\
\hline & & & & Penicillin & $\begin{array}{l}\text { FR ( 14600-fold reduction in } \\
\text { MIC) }\end{array}$ & & \\
\hline & & & & Norfloxacin & FR ( 914-fold reduction in MIC) & & \\
\hline \multirow{10}{*}{ Eugenol } & \multirow{10}{*}{-} & & \multirow{10}{*}{ NCIM 5139} & Ampicillin & \multirow{10}{*}{$\mathrm{CB}(\mathrm{FICI}<0.50)$} & \multirow{10}{*}{ S } & \multirow{10}{*}{ [115] } \\
\hline & & & & Penicillin & & & \\
\hline & & & & Oxacillin & & & \\
\hline & & & & Erythromycin & & & \\
\hline & & & & Norfloxacin & & & \\
\hline & & & & Chloramphenicol & & & \\
\hline & & & & Polymyxin B & & & \\
\hline & & & & Tetracycline & & & \\
\hline & & & & Vancomycin & & & \\
\hline & & & & Rifampin & & & \\
\hline
\end{tabular}


Table 5. Cont.

\begin{tabular}{|c|c|c|c|c|c|c|c|}
\hline $\begin{array}{l}\text { Essential } \\
\text { Oil/Volatile } \\
\text { Compound }\end{array}$ & Vegetal Source & Major Constituent & $\begin{array}{c}\text { Other Gram-negative } \\
\text { Bacteria }\end{array}$ & Antibiotic & Method & Effect & Ref. \\
\hline \multicolumn{8}{|c|}{$\begin{array}{l}\text { Enterobacter cloacae } \\
\text { Strain }\end{array}$} \\
\hline \multirow{4}{*}{ Thyme EO } & \multirow{4}{*}{$\begin{array}{l}\text { Thymi herba (Thymus maroccanus L. } \\
\text { Lamiaceae, Za'ater/Azukeni) }\end{array}$} & \multirow{4}{*}{ Carvacrol (76.35\%) } & \multirow{8}{*}{ Clinical isolate } & Ciprofloxacin & $\mathrm{CB}(\mathrm{FICI}=0.37)$ & \multirow{3}{*}{ S } & \multirow{8}{*}{ [57] } \\
\hline & & & & Gentamicin & $\mathrm{CB}(\mathrm{FICI}=0.19)$ & & \\
\hline & & & & Pristinamycin & $\mathrm{CB}(\mathrm{FICI}=0.50)$ & & \\
\hline & & & & Cefixime & $\mathrm{CB}(\mathrm{FICI}=1.00)$ & I & \\
\hline \multirow{4}{*}{ Thyme EO } & \multirow{4}{*}{$\begin{array}{l}\text { Thymi herba (Thymus broussonetii L. } \\
\text { Lamiaceae, Za'atar Essaouiri) }\end{array}$} & \multirow{4}{*}{$\begin{array}{l}\text { Carvacrol }(39.77 \%) \\
\text { Borneol }(12.03 \%)\end{array}$} & & Ciprofloxacin & $\mathrm{CB}(\mathrm{FICI}=0.50)$ & \multirow{3}{*}{ S } & \\
\hline & & & & Gentamicin & $\mathrm{CB}(\mathrm{FICI}=0.50)$ & & \\
\hline & & & & Pristinamycin & $\mathrm{CB}(\mathrm{FICI}=0.50)$ & & \\
\hline & & & & Cefixime & $\mathrm{CB}(\mathrm{FICI}=1.00)$ & I & \\
\hline \multirow{4}{*}{ Alecrim pimento EO } & \multirow{4}{*}{$\begin{array}{l}\text { Lippiae folium (Lippia sidoides Cham. } \\
\text { Verbenaceae, Alecrim pimento) }\end{array}$} & \multirow{4}{*}{ Thymol (84.9\%) } & \multirow{4}{*}{ ATCC 23355} & Gentamicin & \multirow{4}{*}{ FR (-) } & \multirow{4}{*}{ I } & \multirow{8}{*}{ [102] } \\
\hline & & & & Neomycin & & & \\
\hline & & & & Penicillin G & & & \\
\hline & & & & Ceftriaxone & & & \\
\hline \multirow{4}{*}{ Thymol } & & & \multirow{4}{*}{ ATCC 23355} & Gentamicin & \multirow{4}{*}{ FR (-) } & \multirow{4}{*}{ I } & \\
\hline & - & & & Neomycin & & & \\
\hline & & & & Penicillin G & & & \\
\hline & & & & Ceftriaxone & & & \\
\hline \multicolumn{8}{|c|}{$\begin{array}{l}\text { Proteus vulgaris } \\
\text { Strain }\end{array}$} \\
\hline \multirow{3}{*}{$\alpha$-Bisabolol } & \multirow{3}{*}{$\begin{array}{c}\text { Candeeiro EO } \\
\text { Candeeiro stem Vanillosmopsis arborea } \\
\text { Baker. Asteraceae }\end{array}$} & \multirow{3}{*}{-} & \multirow{3}{*}{ ATCC 13315} & Gentamycin & IZGC (+4\%) & I & \multirow{3}{*}{ [108] } \\
\hline & & & & Tetracycline & IZGC $(-51.72 \%)$ & A & \\
\hline & & & & Tobramycin & IZGC (-53\%) & A & \\
\hline
\end{tabular}


Table 5. Cont.

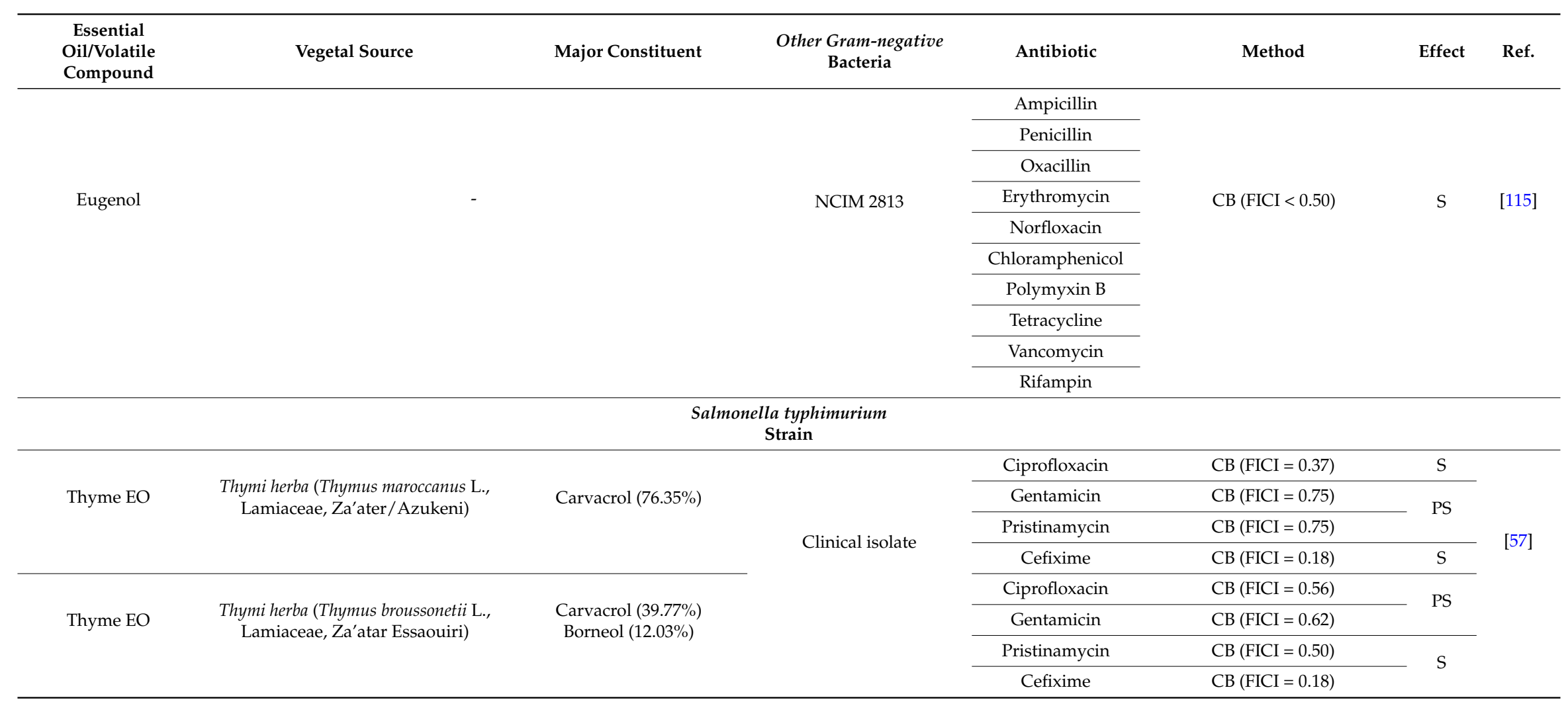


Table 5. Cont.

\begin{tabular}{|c|c|c|c|c|c|c|c|}
\hline $\begin{array}{c}\text { Essential } \\
\text { Oil/Volatile } \\
\text { Compound }\end{array}$ & Vegetal Source & Major Constituent & $\begin{array}{c}\text { Other Gram-negative } \\
\text { Bacteria }\end{array}$ & Antibiotic & Method & Effect & Ref. \\
\hline \multirow{7}{*}{ Carvacrol } & & \multirow{7}{*}{-} & Clinical isolate & Nalidixic acid & $\mathrm{CB}(\mathrm{FICI}=0.31)$ & \multirow{7}{*}{ S } & [124] \\
\hline & & & \multirow{6}{*}{ SGI1 } & Ampicillin & $\mathrm{CB}(\mathrm{FICI}=0.25)$ & & \multirow{12}{*}{ [66] } \\
\hline & & & & Penicillin & $\mathrm{CB}(\mathrm{FICI}=0.37)$ & & \\
\hline & & & & Tetracycline & $\mathrm{CB}(\mathrm{FICI}=0.18)$ & & \\
\hline & & & & Erythromycin & $\mathrm{CB}(\mathrm{FICI}=0.25)$ & & \\
\hline & & & & Bacitracin & $\mathrm{CB}(\mathrm{FICI}=0.25)$ & & \\
\hline & & & & Novobiocin & $\mathrm{CB}(\mathrm{FICI}=0.37)$ & & \\
\hline \multirow{6}{*}{ Eugenol } & & \multirow{6}{*}{-} & \multirow{6}{*}{ SGI1 } & Ampicillin & $\mathrm{CB}(\mathrm{FICI}>0.5)$ & \multirow{2}{*}{ I } & \\
\hline & & & & Penicillin & $\mathrm{CB}(\mathrm{FICI}>0.5)$ & & \\
\hline & & & & Tetracycline & $\mathrm{CB}(\mathrm{FICI}=0.22)$ & $S$ & \\
\hline & & & & Erythromycin & $\mathrm{CB}(\mathrm{FICI}=0.63)$ & \multirow{2}{*}{ I } & \\
\hline & & & & Bacitracin & $\mathrm{CB}(\mathrm{FICI}>0.5)$ & & \\
\hline & & & & Novobiocin & $\mathrm{CB}(\mathrm{FICI}=0.40)$ & $\mathrm{S}$ & \\
\hline \multirow{10}{*}{ Eugenol } & & \multirow{10}{*}{-} & \multirow{10}{*}{ NCIM 2501} & Ampicillin & \multirow{10}{*}{$\mathrm{CB}(\mathrm{FICI}<0.50)$} & \multirow{10}{*}{ S } & \multirow{10}{*}{ [115] } \\
\hline & & & & Penicillin & & & \\
\hline & & & & Oxacillin & & & \\
\hline & & & & Erythromycin & & & \\
\hline & & & & Norfloxacin & & & \\
\hline & & & & Chloramphenicol & & & \\
\hline & & & & Polymyxin B & & & \\
\hline & & & & Tetracycline & & & \\
\hline & & & & Vancomycin & & & \\
\hline & & & & Rifampin & & & \\
\hline
\end{tabular}


Table 5. Cont.

\begin{tabular}{|c|c|c|c|c|c|c|c|}
\hline $\begin{array}{c}\text { Essential Oil/ } \\
\text { Volatile Compound }\end{array}$ & Vegetal Source & Major Constituent & Other Gram-negative Bacteria & Antibiotic & Method & Effect & Ref. \\
\hline \multirow{6}{*}{ Thymol } & & \multirow{6}{*}{ - } & \multirow{6}{*}{ SGI1 } & Ampicillin & $\mathrm{CB}(\mathrm{FICI}=0.12)$ & \multirow{6}{*}{$\mathrm{S}$} & \multirow{12}{*}[66]{} \\
\hline & & & & Penicillin & $\mathrm{CB}(\mathrm{FICI}=0.13)$ & & \\
\hline & & & & Tetracycline & $\mathrm{CB}(\mathrm{FICI}=0.10)$ & & \\
\hline & & & & Erythromycin & $\mathrm{CB}(\mathrm{FICI}=0.25)$ & & \\
\hline & & & & Bacitracin & $\mathrm{CB}(\mathrm{FICI}=0.15)$ & & \\
\hline & & & & Novobiocin & $\mathrm{CB}(\mathrm{FICI}=0.37)$ & & \\
\hline \multirow{6}{*}{ Cinnamaldehyde } & \multirow{6}{*}{\multicolumn{2}{|c|}{-}} & \multirow{6}{*}{ SGI1 } & Ampicillin & $\mathrm{CB}(\mathrm{FICI}=0.25)$ & $\mathrm{S}$ & \\
\hline & & & & Penicillin & $\mathrm{CB}(\mathrm{FICI}=0.63)$ & \multirow{5}{*}{ I } & \\
\hline & & & & Tetracycline & $\mathrm{CB}(\mathrm{FICI}=0.37)$ & & \\
\hline & & & & Erythromycin & $\mathrm{CB}(\mathrm{FICI}=0.24)$ & & \\
\hline & & & & Bacitracin & $\mathrm{CB}(\mathrm{FICI}=0.24)$ & & \\
\hline & & & & Novobiocin & $\mathrm{CB}(\mathrm{FICI}=0.24)$ & & \\
\hline \multirow{6}{*}{ Allyl isothiocyanate } & \multirow{6}{*}{\multicolumn{2}{|c|}{ - }} & \multirow{6}{*}{ SGI1 } & Ampicillin & $\mathrm{CB}(\mathrm{FICI}=0.35)$ & $\mathrm{S}$ & \multirow{6}{*}{ [66] } \\
\hline & & & & Penicillin & $\mathrm{CB}(\mathrm{FICI}=0.63)$ & $\mathrm{I}$ & \\
\hline & & & & Tetracycline & $\mathrm{CB}(\mathrm{FICI}=0.73)$ & & \\
\hline & & & & Erythromycin & $\mathrm{CB}(\mathrm{FICI}=0.48)$ & \multirow{2}{*}{ S } & \\
\hline & & & & Bacitracin & $\mathrm{CB}(\mathrm{FICI}=0.50)$ & & \\
\hline & & & & Novobiocin & $\mathrm{CB}(\mathrm{FICI}=1.00)$ & I & \\
\hline
\end{tabular}

A: antagonism; I: indifference; PS: partial synergy; S: synergy; CB: checkerboard assay; FICI: fractional inhibitory concentration index; FR: fold reduction in MIC; MIC: minimum inhibitory concentration; IZGC (\%): percentage change in inhibition zone diameter due to gaseous contact with essential oil/volatile component. 
Salmonella spp. are motile Gram-negative bacteria. Ninety-nine-point-five percent of the pathogenic Salmonella strains belong to $S$. enterica subsp. enterica. Infections caused by Salmonella are treated with ampicillin, chloramphenicol and trimethoprim-sulfamethoxazole, but multidrug-resistant strains have also been isolated. Fluoroquinolones, extended-spectrum cephalosporins [125] and azithromycin [126] have been used in order to treat multidrug-resistant S. enterica subsp. enterica serovar typhi (S. typhi). Synergistic effects between EOs or volatile compounds and antibiotics against Salmonella strains have been reported (Table 5). The combinations between Thymus maroccanus EO and ciprofloxacin or cefixime, Thymus broussonetii EO and pristinamycin or cefixime exhibited total synergism against Salmonella sp. CCMM B 17 (laboratory collection) [57]. As carvacrol, the main component of both EOs, and nalidixic acid (at low concentrations) target the bacterial membrane; their combination significantly increased the susceptibility of nalidixic acid-resistant Salmonella strains (including S. typhimurium, S. derby, S. enteritidis, S. minnesota) [124]. Both carvacrol and thymol acted synergistically with different antibiotics (ampicillin, penicillin, tetracycline, erythromycin, bacitracin, novobiocin) against tetracycline-resistant $S$. typhimurium SGI, whereas eugenol acted synergistically only with tetracycline and novobiocin [66]. In a study conducted by Hemaiswarya et al. [115], eugenol was found to increase the antibiotic susceptibility of S. typhimurium NCIM 2501. Allyl isothiocyanate (Figure 1), a constituent of EOs isolated from Brassicaceae species [127], acted synergistically in combination with ampicillin, erythromycin and bacitracin against $S$ typhimurium SGI1 [66]. Allyl isothiocyanate targets the same bacterial element as ampicillin and bacitracin: the cell wall [128]. Besides, the central highly electrophile carbon atom of allyl isothiocyanate is a structural pattern that induces the damage of cytoplasmic proteins [104]. Proteic damage caused by allyl isothiocyanate could explain the synergism with erythromycin (inhibitor of protein synthesis through 50S ribosomal subunit binding) [10].

\section{Conclusions}

In conclusion, EOs and their components can enhance the antibiotic activity against Gram-negative bacteria. Most volatile compounds disrupt the bacterial membrane, thus facilitating antibiotic penetration. Enzyme inhibition, reduction of ATP and DNA synthesis and alteration of metabolic pathways are other mechanisms by which EOs and pure volatile compounds act in synergy with conventional antibiotics against Gram-negative bacteria. By inhibiting efflux pumps, volatile compounds, such as geraniol, restore the antibiotic susceptibility of Gram-negative-resistant strains. The mechanisms of synergy of EOs with conventional antibiotics are very complex and still not completely understood as they involve multiple interactions between individual EO constituents, on the one hand, and individual EO constituents and antibiotics, on the other hand. EOs usually possess different antibacterial effects than their major components, which suggests synergistic, additive or antagonistic interactions between their constituents and also an undoubted contribution of minor components to the antibacterial activity. For example, eucalyptus and lavender EOs showed higher antibacterial effects against Staphylococcus aureus, Bacillus subtilis and Escherichia coli compared to their major components, 1,8-cineole and linalyl acetate, respectively. On the contrary, thyme EO was less active against the same bacterial strains than its main constituent, thymol [129]. Both thymol and carvacrol have been detected in EOs isolated from certain Lippia, Thymus and Origanum species [57,76,107,130-133]. Surprisingly, these two monoterpene phenols might modulate the antibiotic activity in a different manner. For instance, thymol showed excellent synergistic effects with penicillin against Escherichia coli (resistant to some $\beta$-lactam antibiotics), whereas the combination of carvacrol with penicillin resulted in an indifferent effect [65]. The complexity of interactions generated when combining EOs and antibiotics is evident. Overall, the results of the in vitro assays are very promising for the development of new effective formulations against Gram-negative bacteria. Obviously, further in vivo studies are required to evaluate the bioavailability, efficacy and toxicity of $\mathrm{EO} /$ volatile compound-antibiotic combinations. 
Author Contributions: P.A. and A.M. conceived and designed the review protocol, contributed to literature search and writing of the manuscript, corrected the drafts and final version of the manuscript. A.T., A.B., E.G. and A.C.A. contributed to literature search and writing of the manuscript.

Conflicts of Interest: The authors declare no conflict of interest.

\section{References}

1. Abat, C.; Chaudet, H.; Rolain, J.M.; Colson, P.; Raoult, D. Traditional and syndromic surveillance of infectious diseases and pathogens. Int. J. Infect. Dis. 2016, 48, 22-28. [CrossRef] [PubMed]

2. Van Vuuren, S.F.; Suliman, S.; Viljoen, A.M. The antimicrobial activity of four commercial essential oils in combination with conventional antimicrobials. Lett. Appl. Microbiol. 2009, 48, 440-446. [CrossRef] [PubMed]

3. Summary of the Latest Data on Antibiotic Resistance in the European Union. Available online: http: / / ecdc.europa.eu/en/eaad/Documents/antibiotics-resistance-EU-data-2013.pdf (accessed on 10 June 2016).

4. Wolska, K.I.; Grześ, K.; Kurek, A. Synergy between novel antimicrobials and conventional antibiotics or bacteriocins. Pol. J. Microbiol. 2012, 61, 95-104. [PubMed]

5. Elshafie, H.S.; Mancini, E.; Sakr, S.; de Martino, L.; Mattia, C.A.; de Feo, V.; Camele, I. Antifungal activity of some constituents of Origanum vulgare L. essential oil against postharvest disease of peach fruit. J. Med. Food 2015, 18, 929-934. [CrossRef] [PubMed]

6. Mancini, E.; Camele, I.; Elshafie, H.S.; de Martino, L.; Pellegrino, C.; Grulova, D.; de Feo, V. Chemical composition and biological activity of the essential oil of Origanum vulgare ssp. hirtum from different areas in the Southern Apennines (Italy). Chem. Biodivers. 2014, 11, 639-651. [CrossRef] [PubMed]

7. Van Vuuren, S.; Viljoen, A. Plant-based antimicrobial studies-Methods and approaches to study the interaction between natural products. Planta Med. 2011, 77, 1168-1182. [CrossRef] [PubMed]

8. Bassolé, I.H.; Juliani, H.R. Essential oils in combination and their antimicrobial properties. Molecules 2012, 17, 3989-4006. [CrossRef] [PubMed]

9. Basri, D.F.; Xian, L.W.; Abdul Shukor, N.I.; Latip, J. Bacteriostatic antimicrobial combination: Antagonistic interaction between epsilon-viniferin and vancomycin against methicillin-resistant Staphylococcus aureus. Biomed. Res. Int. 2014. [CrossRef] [PubMed]

10. Langeveld, W.T.; Veldhuizen, E.J.; Burt, S.A. Synergy between essential oil components and antibiotics: A review. Crit. Rev. Microbiol. 2014, 40, 76-94. [CrossRef] [PubMed]

11. Kyaw, B.M.; Arora, S.; Lim, C.S. Bactericidal antibiotic-phytochemical combinations against methicillin resistant Staphylococcus aureus. Braz. J. Microbiol. 2012, 43, 938-945. [CrossRef] [PubMed]

12. Moon, S.E.; Kim, H.Y.; Cha, J.D. Synergistic effect between clove oil and its major compounds and antibiotics against oral bacteria. Arch. Oral. Biol. 2011, 56, 907-916. [CrossRef] [PubMed]

13. Odds, F.C. Synergy, antagonism, and what the chequerboard puts between them. J. Antimicrob. Chemother. 2003. [CrossRef] [PubMed]

14. Hendry, E.R.; Worthington, T.; Conway, B.R.; Lambert, P.A. Antimicrobial efficacy of eucalyptus oil and 1,8-cineole alone and in combination with chlorhexidine digluconate against microorganisms grown in planktonic and biofilm cultures. J. Antimicrob. Chemother. 2009, 64, 1219-1225. [CrossRef] [PubMed]

15. Gibbons, S. Phytochemicals for bacterial resistance-Strengths, weaknesses and opportunities. Planta Med. 2008, 74, 594-602. [CrossRef] [PubMed]

16. Kourtesi, C.; Ball, A.R.; Huang, Y.Y.; Jachak, S.M.; Vera, D.M.; Khondkar, P.; Gibbons, S.; Hamblin, M.R.; Tegos, G.P. Microbial efflux systems and inhibitors: Approaches to drug discovery and the challenge of clinical implementation. Open Microbiol. J. 2013, 7, 34-52. [CrossRef] [PubMed]

17. Garvey, M.I.; Rahman, M.M.; Gibbons, S.; Piddock, L.J. Medicinal plant extracts with efflux inhibitory activity against Gram-negative bacteria. Int. J. Antimicrob. Agents 2011, 37, 145-151. [CrossRef] [PubMed]

18. Hemaiswarya, S.; Kruthiventi, A.K.; Doble, M. Synergism between natural products and antibiotics against infectious diseases. Phytomedicine 2008, 15, 639-652. [CrossRef] [PubMed]

19. Cole, E.R.; dos Santos, R.B.; Lacerda Júnior, V.; Martins, J.; Greco, S.; Cunha Neto, A. Chemical composition of essential oil from ripe fruit of Schinus terebinthifolius raddi and evaluation of its activity against wild strains of hospital origin. Braz. J. Microbiol. 2014, 45, 821-828. [CrossRef] [PubMed] 
20. Elshafie, H.S.; Ghanney, N.; Mang, S.M.; Ferchichi, A.; Camele, I. An in vitro attempt for controlling severe phytopathogens and human pathogens using essential oils from mediterranean plants of genus schinus. J. Med. Food 2016, 19, 266-273. [CrossRef] [PubMed]

21. Howard, A.; O'Donoghue, M.; Feeney, A.; Sleator, R.D. Acinetobacter baumannii: An emerging opportunistic pathogen. Virulence 2012, 3, 243-250. [CrossRef] [PubMed]

22. Tripathi, P.C.; Gajbhiye, S.R.; Agrawal, G.N. Clinical and antimicrobial profile of Acinetobacter spp.: An emerging nosocomial superbug. Adv. Biomed. Res. 2014. [CrossRef] [PubMed]

23. Peleg, A.Y.; Seifert, H.; Paterson, D.L. Acinetobacter baumannii: Emergence of a successful pathogen. Clin. Microbiol. Rev. 2008, 21, 538-582. [CrossRef] [PubMed]

24. Aleksic, V.; Dukic, N.M.; Simin, N.; Nedeljkovic, N.S.; Knezevic, P. Synergistic effect of Myrtus communis L. essential oils and conventional antibiotics against multi-drug resistant Acinetobacter baumannii wound isolates. Phytomedicine 2014, 21, 1666-1674. [CrossRef] [PubMed]

25. Knezevic, P.; Aleksic, V.; Simin, N.; Svircev, E.; Petrovic, A.; Mimica-Dukic, N. Antimicrobial activity of Eucalyptus camaldulensis essential oils and their interactions with conventional antimicrobial agents against multi-drug resistant Acinetobacter baumannii. J. Ethnopharmacol. 2016, 178, 125-136. [CrossRef] [PubMed]

26. Jacobs, A.C.; Hood, I.; Boyd, K.L.; Olson, P.D.; Morrison, J.M.; Carson, S.; Sayood, K.; Iwen, P.C.; Skaar, E.P.; Dunman, P.M. Inactivation of phospholipase D diminishes Acinetobacter baumannii pathogenesis. Infect. Immun. 2010, 78, 1952-1962. [CrossRef] [PubMed]

27. Camarena, L.; Bruno, V.; Euskirchen, G.; Poggio, S.; Snyder, M. Molecular mechanisms of ethanol-induced patho-genesis revealed by RNA-sequencing. PLoS Pathog. 2010. [CrossRef] [PubMed]

28. Munoz-Price, S.; Weinstein, R.A. Acinetobacter Infection. N. Engl. J. Med. 2008, 358, 1271-1281. [CrossRef] [PubMed]

29. Doi, Y.; Husain, S.; Potoski, B.A.; McCurry, K.R.; Paterson, D.L. Extensively drug-resistant Acinetobacter baumannii. Emerg. Infect. Dis. 2009, 15, 980-982. [CrossRef] [PubMed]

30. Duarte, A.; Ferreira, S.; Silva, F.; Domingues, F.C. Synergistic activity of coriander oil and conventional antibiotics against Acinetobacter baumannii. Phytomedicine 2012, 19, 236-238. [CrossRef] [PubMed]

31. Silva, F.; Ferreira, S.; Queiroz, J.A.; Domingues, F.C. Coriander (Coriandrum sativum L.) essential oil: Its antibacterial activity and mode of action evaluated by flow cytometry. J. Med. Microbiol. 2011, 60, 1479-1486. [CrossRef] [PubMed]

32. Prabuseenivasan, S.; Jayakumar, M.; Ignacimuthu, S. In vitro antibacterial activity of some plant essential oils. BMC Complement. Altern. Med. 2006. [CrossRef] [PubMed]

33. Zaika, L.L. Spices and herbs: Their antibacterial activity and its determination. J. Food Saf. 1988, 23, 97-118.

34. Rosato, A.; Piarulli, M.; Corbo, F.; Muraglia, M.; Carone, A.; Vitali, M.E.; Vitali, C. In vitro synergistic antibacterial action of certain combinations of gentamicin and essential oils. Curr. Med. Chem. 2010, 17, 3289-3295. [CrossRef] [PubMed]

35. Tam, V.H.; Schilling, A.N.; Vo, G.; Kabbara, S.; Kwa, A.L.; Wiederhold, N.P.; Lewis, R.E. Pharmacodynamics of polymyxin B against Pseudomonas aeruginosa. Antimicrob. Agents Chemother. 2005, 49, 3624-3630. [CrossRef] [PubMed]

36. Lopez-Romero, J.C.; González-Ríos, H.; Borges, A.; Simões, M. Antibacterial effects and mode of action of selected essential oils components against Escherichia coli and Staphylococcus aureus. Evid. Based Complement. Altern. Med. 2015. [CrossRef] [PubMed]

37. Lorenzi, V.; Muselli, A.; Bernardini, A.F.; Berti, L.; Pagès, J.M.; Amaral, L.; Bolla, J.M. Geraniol restores antibiotic activities against multidrug-resistant isolates from Gram-negative species. Antimicrob. Agents Chemother. 2009, 53, 2209-2211. [CrossRef] [PubMed]

38. Azevedo, M.M.; Ramalho, P.; Silva, A.P.; Teixeira-Santos, R.; Pina-Vaz, C.; Rodrigues, A.G. Polyethyleneimine and polyethyleneimine-based nanoparticles: Novel bacterial and yeast biofilm inhibitors. J. Med. Microbiol. 2014, 63, 1167-1173. [CrossRef] [PubMed]

39. Li, L.; Li, Z.W.; Yin, Z.Q.; Wei, Q.; Jia, R.Y.; Zhou, L.J.; Xu, J.; Song, X.; Zhou, Y.; Du, Y.H.; et al. Antibacterial activity of leaf essential oil and its constituents from Cinnamomum longepaniculatum. Int. J. Clin. Exp. Med. 2014, 7, 1721-1727. [PubMed]

40. Guerra, F.Q.; Mendes, J.M.; Sousa, J.P.; Morais-Braga, M.F.; Santos, B.H.; Coutinho, M.H.D.; Lima, E.O. Increasing antibiotic activity against a multidrug-resistant Acinetobacter spp. by essential oils of Citrus limon and Cinnamomum zeylanicum. Nat. Prod. Res. 2012, 26, 2235-2238. [CrossRef] [PubMed] 
41. Darfeuille-Michaud, A.; Boudeau, J.; Bulois, P.; Neut, C.; Glasser, A.L.; Barnich, N.; Bringer, M.A.; Swidsinski, A.; Beaugerie, L.; Colombel, J.F. High prevalence of adherent-invasive Escherichia coli associated with ileal mucosa in Crohn's disease. Gastroenterology 2004, 127, 412-421. [CrossRef] [PubMed]

42. Allocati, N.; Masulli, M.; Alexeyev, M.F.; di Ilio, C. Escherichia coli in Europe: An overview. Int. J. Environ. Res. Public Health 2013, 10, 6235-6254. [CrossRef] [PubMed]

43. Croxen, M.A.; Finlay, B.B. Molecular mechanisms of Escherichia coli pathogenicity. Nat. Rev. Microbiol. 2010, 8, 26-38. [PubMed]

44. Johnson, J.R.; Stell, A.L. Extended virulence genotypes of Escherichia coli strains from patients with urosepsis in relation to phylogeny and host compromise. J. Infect. Dis. 2000, 181, 261-272. [CrossRef] [PubMed]

45. Kaper, J.B.; Nataro, J.P.; Mobley, H.L. Pathogenic Escherichia coli. Nat. Rev. Microbiol. 2004, 2, $123-140$. [CrossRef] [PubMed]

46. Qadri, F.; Svennerholm, A.M.; Faruque, A.S.; Sack, R.B. Enterotoxigenic Escherichia coli in developing countries: Epidemiology, microbiology, clinical features, treatment, and prevention. Clin. Microbiol. Rev. 2005, 18, 465-483. [CrossRef] [PubMed]

47. Poirel, L.; Bonnin, R.A.; Nordmann, P. Genetic support and diversity of acquired extended-spectrum $\beta$-lactamases in Gram-negative rods. Infect. Genet. Evol. 2012, 12, 883-893. [CrossRef] [PubMed]

48. Queenan, A.M.; Bush, K. Carbapenemases: The versatile beta-lactamases. Clin. Microbiol. Rev. 2007, 20, 440-458. [CrossRef] [PubMed]

49. Robicsek, A.; Jacoby, G.A.; Hooper, D.C. The worldwide emergence of plasmid-mediated quinolone resistance. Lancet Infect. Dis. 2006, 6, 629-640. [CrossRef]

50. Si, H.; Hu, J.; Liu, Z.; Zeng, Z.L. Antibacterial effect of oregano essential oil alone and in combination with antibiotics against extended-spectrum beta-lactamase-producing Escherichia coli. FEMS Immunol. Med. Microbiol. 2008, 53, 190-194. [CrossRef] [PubMed]

51. Okusu, H.; Ma, D.; Nikaido, H. AcrAB efflux pump plays a major role in the antibiotic resistance phenotype of Escherichia coli multiple-antibiotic-resistance (Mar) mutants. J. Bacteriol. 1996, 178, 306-308. [PubMed]

52. Fadli, M.; Chevalier, J.; Saad, A.; Mezrioui, N.E.; Hassani, L.; Pagès, J.M. Essential oils from Moroccan plants as potential chemosensitisers restoring antibiotic activity in resistant Gram-negative bacteria. Int. J. Antimicrob. Agents 2011, 38, 325-330. [CrossRef] [PubMed]

53. Xu, J.; Zhou, F.; Ji, B.P.; Pei, R.S.; Xu, N. The antibacterial mechanism of carvacrol and thymol against Escherichia coli. Lett. Appl. Microbiol. 2008, 47, 174-179. [CrossRef] [PubMed]

54. Burt, S. Essential oils: Their antibacterial properties and potential applications in foods-A review. Int. J. Food Microbiol. 2004, 94, 223-253. [CrossRef] [PubMed]

55. Helander, I.M.; Alakomi, H.L.; Latva-Kala, K.; Mattila-Sandholm, T.; Pol, I.; Smid, E.J.; Gorris, L.G.M.; von Wright, A. Characterization of the action of selected essential oil components on Gram-negative bacteria. J. Agric. Food Chem. 1998, 46, 3590-3595. [CrossRef]

56. Ultee, A.; Bennik, M.H.; Moezelaar, R. The phenolic hydroxyl group of carvacrol is essential for action against the food-borne pathogen Bacillus cereus. Appl. Environ. Microbiol. 2002, 68, 1561-1568. [CrossRef] [PubMed]

57. Fadli, M.; Saada, A.; Sayadib, S.; Chevalierc, J.; Mezriouia, N.E.; Pagès, J.M.; Hassani, L. Antibacterial activity of Thymus maroccanus and Thymus broussonetii essential oils against nosocomial infection-bacteria and their synergistic potential with antibiotics. Phytomedicine 2012, 19, 464-471. [CrossRef] [PubMed]

58. Fadli, M.; Bollab, J.M.; Mezriouia, N.E.; Pagès, J.M.; Hassania, L. First evidence of antibacterial and synergistic effects of Thymus riatarum essential oil with conventional antibiotics. Ind. Crop. Prod. 2014, 61, 370-376. [CrossRef]

59. Guinoiseau, E.; Lorenzi, V.; Luciani, A.; Muselli, A.; Costa, J.; Casanova, J.; Berti, L. Biological properties and resistance reversal effect of Helichrysum italicum (Roth) G. Don. Formatex 2013, 2, 1073-1080.

60. Miladinović, D.L.; Ilić, B.S.; Kocić, B.D.; Miladinović, M.D. An in vitro antibacterial study of savory essential oil and geraniol in combination with standard antimicrobials. Nat. Prod. Commun. 2014, 9, 1629-1632. [PubMed]

61. Barreto, H.M.; Silva Filho, E.C.; Lima, E.D.O.; Coutinho, H.D.M.; Morais Braga, M.F.B.; Tavares, C.C.A.; Tintinoc, S.R.; Rego, J.V.; de Abreu, A.P.L.; Lustosa Mdo, C.; et al. Chemical composition and possible use as adjuvant of the antibiotic therapy of the essential oil of Rosmarinus officinalis L. Ind. Crop. Prod. 2014, 59, 290-294. [CrossRef] 
62. Costa-Lotufo, L.V.; Araújo, E.C.; Lima, M.A.; Moraes, M.E.; Pessoa, C.; Silviera, E.R.; Moraes, M.O. Antiproliferative effects of abietane diterpenoids isolated from Hyptis martiusii Benth (Labiatae). Pharmazie 2004, 59, 78-79. [PubMed]

63. Caldas, G.F.; do Amaral Costa, I.M.; da Silva, J.B.; da Nóbrega, R.F.; Rodrigues, F.F.; da Costa, J.G.; Wanderley, A.G. Antiulcerogenic activity of the essential oil of Hyptis martiusii Benth. (Lamiaceae). J. Ethnopharmacol. 2011, 137, 886-892. [CrossRef] [PubMed]

64. De Oliveira, A.D.; Galvao Rodrigue, F.F.; Coutinho, D.M.H.; da Costa, J.G.; de Menezes, I.R. Chemical composition, modulatory bacterial resistance and antimicrobial activity of essential oil the Hyptis martiusii benth. by direct and gaseous contact. Jundishapur J. Nat. Pharm. Prod. 2014, 9, e13521. [CrossRef] [PubMed]

65. Gallucci, M.N.; Casero, C.; de las Mercedes, M.; Demo, M.S. Interaction between terpenes and penicillin on bacterial strains resistant to beta-lactam antibiotics. Mol. Med. Chem. 2006, 10, 30-32.

66. Palaniappan, K.; Holley, R.A. Use of natural antimicrobials to increase antibiotic susceptibility of drug resistant bacteria. Int. J. Food. Microbiol. 2010, 140, 164-168. [CrossRef] [PubMed]

67. Ben Arfa, A.; Combes, S.; Preziosi-Belloy, L.; Gontard, N.; Chalier, P. Antimicrobial activity of carvacrol related to its chemical structure. Lett. Appl. Microbiol. 2006, 43, 149-154. [CrossRef] [PubMed]

68. Di Pasqua, R.; Hoskins, N.; Betts, G.; Mauriello, G. Changes in membrane fatty acids composition of microbial cells induced by addiction of thymol, carvacrol, limonene, cinnamaldehyde, and eugenol in the growing media. J. Agric. Food Chem. 2006, 54, 2745-2749. [CrossRef] [PubMed]

69. Novak, J.; Zitterl-Eglseer, K.; Deans, S.G.; Franz, C.M. Essential oils of different cultivars of Cannabis sativa L. and their antimicrobial activity. Flavour Fragr. J. 2001, 16, 259-262. [CrossRef]

70. Bernotienë, G.; Nivinskienë, O.; Butkienë, R.; Mockutë, D. Chemical composition of essential oil of hops (Humulus lupulus L.) growing in Aukstaitija. Chemija 2004, 2, 31-36.

71. Alves, R.J.V.; Medeiros, D.; de Moura, R.L.; de Gusmão, L.C.T.; da Silva, N.G.; Antunes, K.; Matozinhos, C.; Blanco, M.A. First report of naturalization of Houttuynia cordata Thunb. 1783 (Piperales: Saururaceae) in South America. Check List 2013, 9, 642-644. [CrossRef]

72. Dai, D.N.; Thang, T.D.; Ogunmoye, A.R.; Olanrewaju, I.A.; Ogunwande, I.A. Chemical constituents of essential oils from the leaves of Tithonia diversifolia, Houttuynia cordata and Asarum glabrum grown in Vietnam. Am. J. Essent. Oils Nat. Prod. 2015, 2, 17-21.

73. Tajidin, N.E.; Ahmad, S.H.; Rosenani, A.B.; Azimah, H.; Munirah, M. Chemical composition and citral content in lemongrass (Cymbopogon citratus) essential oil at three maturity stages. Afr. J. Biotechnol. 2012, 7, 2685-2693. [CrossRef]

74. Miladinović, D.L.; Ilic, B.S.; Miladinovic, L.C.; Kocic, B.D.; Ciric, V.M.; Stankov-Jovanovic, V.P.; Cvetkovic, O.G. Antibacterial activity of Thymus pulegioides essential oil and its synergistic potential with antibiotics: A chemometric approach. In Recent Progress in Medicinal Plants, Essential Oils III and Phytopharmacology; Govil, J.N., Bhattacharya, S., Eds.; Studium Press LLC: Houston, TX, USA, 2013; pp. 101-136.

75. Djouahri, A.; Saka, B.; Boudarene, L.; Benseradj, L.; Aberrane, S.; Aitmoussa, S.; Chelghoum, C.; Lamari, L.; Sabaou, L.; Baaliouame, A. In vitro synergistic/antagonistic antibacterial and anti-inflammatory effect of various extracts/essential oil from cones of Tetraclinis articulata (Vahl) Masters with antibiotic and anti-inflammatory agents. Ind. Crop. Prod. 2014, 56, 60-66. [CrossRef]

76. Kasrati, A.; Jamall, C.A.; Fadli, M.; Bekkouche, K.; Hassani, L.; Wohlmuth, H.; Leach, D.; Abbad, A. Antioxidative activity and synergistic effect of Thymus saturejoides Coss. essential oils with cefixime against selected food-borne bacteria. Ind. Crop. Prod. 2014, 61, 338-344. [CrossRef]

77. Brisse, S.; Grimont, F.; Grimont, P.A.D. The Genus Klebsiella. Prokaryotes 2006, 6, 159-196.

78. Podschun, R.; Ullmann, U. Klebsiella spp. as nosocomial pathogens: Epidemiology, taxonomy, typing methods and pathogenicity factors. Clin. Microbiol. Rev. 1998, 11, 589-603. [PubMed]

79. Fung, C.P.; Chang, F.Y.; Lee, S.C.; Hu, B.S.; Kuo, B.I.; Liu, C.Y.; Ho, M.; Siu, L.K. A global emerging disease of Klebsiella pneumoniae liver abscess: Is serotype $\mathrm{K} 1$ an important factor for complicated endophthalmitis? Gut 2002, 50, 420-424. [CrossRef] [PubMed]

80. Lin, W.H.; Kao, C.Y.; Yang, D.C.; Tseng, C.C.; Wu, A.B.; Teng, C.H.; Wang, M.C.; Wu, J.J. Clinical and microbiological characteristics of Klebsiella pneumoniae from community-acquired recurrent urinary tract infections. Eur. J. Clin. Microbiol. Infect. Dis. 2014, 33, 1533-1539. [CrossRef] [PubMed] 
81. Ko, W.C.; Paterson, D.L.; Sagnimeni, A.J.; Hansen, D.S.; von Gottberg, A.; Mohapatra, S.; Casellas, J.M.; Goossens, H.; Mulazimoglu, L.; Trenholme, G.; et al. Community acquired Klebsiella pneumoniae bacteremia: Global differences in clinical patterns. Emerg. Infect. Dis. 2002, 8, 160-166. [CrossRef] [PubMed]

82. Li, B.; Zhao, Y.; Liu, C.; Chen, Z.; Zhou, D. Molecular pathogenesis of Klebsiella pneumoniae. Future Microbiol. 2014, 9, 1071-1081. [CrossRef] [PubMed]

83. Vuotto, C.; Longo, F.; Balice, M.P.; Donelli, G.; Varaldo, P.E. Antibiotic resistance related to biofilm formation in Klebsiella pneumoniae. Pathogens 2014, 3, 743-758. [CrossRef] [PubMed]

84. Nordmann, P.; Cuzon, G.; Naas, T. The real threat of Klebsiella pneumoniae carbapenemase-producing bacteria. Lancet Infect. Dis. 2009, 9, 228-236. [CrossRef]

85. Petrosillo, N.; Giannella, M.; Lewis, R.; Viale, P. Treatment of carbapenem-resistant Klebsiella pneumoniae: The state of the art. Expert Rev. Anti Infect. Ther. 2013, 11, 159-177. [CrossRef] [PubMed]

86. Munoz-Price, L.S.; Poirel, L.; Bonomo, R.A.; Schwaber, M.J.; Daikos, G.L.; Cormican, M.; Cornaglia, G.; Garau, J.; Gniadkowski, M.; Hayden, M.K.; et al. Clinical epidemiology of the global expansion of Klebsiella pneumoniae carbapenemases. Lancet Infect. Dis. 2013, 13, 785-796. [CrossRef]

87. Parisi, S.G.; Bartolini, A.; Santacatterina, E.; Castellani, E.; Ghirardo, R.; Berto, A.; Franchin, E.; Menegotto, N.; de Canale, E.; Tommasini, T.; et al. Prevalence of Klebsiella pneumoniae strains producing carbapenemases and increase of resistance to colistin in an Italian teaching hospital from January 2012 to December 2014. BMC Infect. Dis. 2015. [CrossRef] [PubMed]

88. Qureshi, Z.A.; Paterson, D.L.; Potoski, B.A.; Kilayko, M.C.; Sandovsky, G.; Sordillo, E.; Polsky, B.; Adams-Haduch, J.M.; Doi, Y. Treatment outcome of bacteremia due to KPC-producing Klebsiella pneumoniae: Superiority of combination antimicrobial regimens. Antimicrob. Agents Chemother. 2012, 56, 2108-2113. [CrossRef] [PubMed]

89. Ultee, A.; Kets, E.P.W.; Smid, E.J. Mechanisms of action of carvacrol on the food-borne pathogen Bacillus cereus. Appl. Environ. Microbiol. 1999, 65, 4606-4610. [PubMed]

90. Miladinović, D.L.; Ilić, B.S.; Mihajilov-Krstev, T.M.; Jović, J.L.; Marković, MS. In vitro antibacterial activity of Libanotis montana essential oil in combination with conventional antibiotics. Nat. Prod. Commun. 2014, 9, 281-286. [PubMed]

91. Sikkema, J.; de Bont, J.A.; Poolman, B. Mechanisms of membrane toxicity of hydrocarbons. Microbiol. Rev. 1995, 59, 201-222. [PubMed]

92. Yap, P.S.X.; Yiap, B.C.; Ping, H.C.; Lim, S.H.E. Essential oils, a new horizon in combating bacterial antibiotic resistance. Open Microbiol. J. 2014, 8, 6-14. [CrossRef] [PubMed]

93. Moore, N.M.; Flaws, M.L. Antimicrobial resistance mechanisms in Pseudomonas aeruginosa. Clin. Lab. Sci. 2011, 24, 47-51. [PubMed]

94. Fujitani, S.; Sun, H.Y.; Yu, V.L.; Weingarten, J.A. Pneumonia due to Pseudomonas aeruginosa: Part I: epidemiology, clinical diagnosis and source. Chest 2011, 139, 909-919. [CrossRef] [PubMed]

95. Mesaros, N.; Nordmann, P.; Plésiat, P.; Roussel-Delvallez, M.; van Eldere, J.; Glupczynski, Y.; van Laethem, Y.; Jacobs, F.; Lebecque, P.; Malfroot, A.; et al. Pseudomonas aeruginosa: Resistance and therapeutic options at the turn of the new millennium. Clin. Microbiol. Infect. 2007, 13, 560-578. [CrossRef] [PubMed]

96. Juan, C.; Maciá, M.D.; Gutiérrez, O.; Vidal, C.; Pérez, J.L.; Oliver, A. Molecular mechanisms of $\beta$-lactam resistance mediated by AmpC hyperproduction in Pseudomonas aeruginosa clinical strains. Antimicrob. Agents Chemother. 2005, 49, 4733-4738. [CrossRef] [PubMed]

97. Ben Haj Khalifa, A.; Moissenet, D.; vu Thien, H.; Khedher, M. Virulence factors in Pseudomonas aeruginosa: Mechanisms and modes of regulation. Ann. Biol. Clin. 2011, 69, 393-403.

98. Lister, P.D.; Wolter, D.J.; Hanson, N.D. Antibacterial-resistant Pseudomonas aeruginosa: Clinical impact and complex regulation of chromosomally encoded resistance mechanisms. Clin. Microbiol. Rev. 2009, 22, 582-610. [CrossRef] [PubMed]

99. Silva, A.V.; da Sousa, P.J.; Pessôa, L.F.H.; de Freitas, F.R.A.; Coutinho, D.M.H.; Alves, B.N.L.; Lima, O.E. Ocimum basilicum: Antibacterial activity and association study with antibiotics against bacteria of clinical importance. Pharm. Biol. 2015, 54, 863-867. [CrossRef] [PubMed]

100. Kohanski, M.A.; Dwyer, D.J.; Collins, J.J. How antibiotics kill bacteria: From targets to networks. Nat. Rev. Microbiol. 2010, 8, 423-435. [CrossRef] [PubMed]

101. El-Hosseiny, L.; el-Shenawy, M.; Haroun, M.; Abdullah, F. Comparative evaluation of the inhibition effect of some essential oils with antibiotics against Pseudomonas aeruginosa. Int. J. Antibiot. 2014. [CrossRef] 
102. Veras, H.N.H.; Rodrigues, F.G.F.; Botelho, M.A.; Menezes, I.R.A.; Coutinho, H.D.M.; Costa, J.G.M. Enhancement of aminoglycosides and $\beta$-lactams antibiotic activity by essential oil of Lippia sidoides Cham. and the Thymol. Arab. J. Chem. 2013. [CrossRef]

103. Faleiro, M.L. The mode of antibacterial action of essential oils. In Science against Microbial Pathogens: Communicating Current Research and Technological Advances; Méndez Vilas, A., Ed.; Formatex Research Center: Badajoz, Spain, 2011; pp. 1143-1156.

104. Hyldgaard, M.; Mygind, T.; Meyer, R.L. Essential oils in food preservation: Mode of action, synergies, and interactions with food matrix components. Front. Microbiol. 2012. [CrossRef] [PubMed]

105. Griffin, S.G.; Wyllie, S.G.; Markham, J.L.; Leach, D.N. The role of structure and molecular properties of terpenoids in determining their antimicrobial activity. Flavour Fragr. J. 1999, 14, 322-332. [CrossRef]

106. Rodrigues, F.F.; Costa, J.G.; Coutinho, H.D. Enhancement of the antibiotic activity of gentamicin by volatile compounds of Zanthoxylum articulatum. Indian J. Med. Res. 2010, 131, 833-835.

107. Bitu, V.C.; Fecundo, H.D.; da Costa, J.G.; Coutinho, H.D.; Rodrigues, F.F.; de Santana, N.M.; Botelho, M.A.; Menezes, I.R. Chemical composition of the essential oil of Lippia gracilis schauer leaves and its potential as modulator of bacterial resistance. Nat. Prod. Res. 2014, 28, 399-402. [CrossRef] [PubMed]

108. Santos, N.K.A.; Rodrigues, F.F.G.; Coutinho, H.D.M.; Viana, G.S.B.; Costa, J.G.M. Isolation of alpha-bisabolol from the essential oil of Vanillosmopsis arborea baker and modulation of antibiotic activity using gaseous contact. J. Essent. Oil Bear. Plants 2013, 16, 826-831. [CrossRef]

109. Rodrigues, F.F.; Costa, J.G.; Coutinho, H.D. Synergy effects of the antibiotics gentamicin and the essential oil of Croton zehntneri. Phytomedicine 2009, 16, 1052-1055. [CrossRef] [PubMed]

110. Hilmer, S.N.; Tran, K.; Rubie, P.; Wright, J.; Gnjidic, D.; Mitchell, S.J.; Matthews, S. Gentamicin pharmacokinetics in old age and frailty. Br. J. Clin. Pharmacol. 2011, 71, 224-231. [CrossRef] [PubMed]

111. Brehm-Stecher, B.F.; Johnson, E.A. Sensitization of Staphylococcus aureus and Escherichia coli to antibiotics by the sesquiterpenoids nerolidol, farnesol, bisabolol, and apritone. Antimicrob. Agents Chemother. 2003, 47, 3357-3360. [CrossRef] [PubMed]

112. Coutinho, H.D.M.; Rodrigues, F.F.G.; Nascimento, E.M.M.; Costa, J.M.C.; Falcão-Silva, V.S.; Siqueira-Júnior, J.P. Synergism of gentamicin and norfloxacin with the volatile compounds of Lippia microphylla cham. (Verbenaceae). J. Essent. Oil Res. 2011, 23, 24-28.

113. De Sousa, E.O.; Rodrigues, F.F.; Campos, A.R.; Lima, S.G.; da Costa, J.G. Chemical composition and synergistic interaction between aminoglycosides antibiotics and essential oil of Lantana montevidensis Briq. Nat. Prod. Res. 2013, 27, 942-945. [CrossRef] [PubMed]

114. Dahham, S.S.; Tabana, Y.M.; Iqbal, M.A.; Ahamed, M.B.; Ezzat, M.O.; Majid, A.S.; Majid, A.M. The anticancer, antioxidant and antimicrobial properties of the sesquiterpene $\beta$-caryophyllene from the essential oil of Aquilaria crassna. Molecules 2015, 20, 11808-11829. [CrossRef] [PubMed]

115. Hemaiswarya, S.; Doble, M. Synergistic interaction of eugenol with antibiotics against Gram negative bacteria. Phytomedicine 2009, 16, 997-1005. [CrossRef] [PubMed]

116. Thiolas, A.; Bollet, C.; la Scola, B.; Raoult, D.; Pagès, J.M. Successive emergence of Enterobacter aerogenes strains resistant to imipenem and colistin in a patient. Antimicrob. Agents Chemother. 2005, 49, 1354-1358. [CrossRef] [PubMed]

117. Davin-Regli, A.; Pagès, J.M. Enterobacter aerogenes and Enterobacter cloacae; versatile bacterial pathogens confronting antibiotic treatment. Front. Microbiol. 2015. [CrossRef] [PubMed]

118. Lavigne, J.P.; Sotto, A.; Nicolas-Chanoine, M.H.; Bouziges, N.; Bourg, G.; Davin-Regli, A.; Pagès, J.M. Membrane permeability, a pivotal function involved in antibiotic resistance and virulence in Enterobacter aerogenes clinical isolates. Clin. Microbiol. Infect. 2012, 18, 539-545. [CrossRef] [PubMed]

119. Fadli, M.; Chevalier, J.; Bolla, J.M.; Mezrioui, N.E.; Hassani, L.; Pagès, J.M. Thymus maroccanus essential oil, a membranotropic compound active on Gram-negative bacteria and resistant isolates. J. Appl. Microbiol. 2012, 113, 1120-1129. [CrossRef] [PubMed]

120. Campos, M.A.; Morey, P.; Bengoechea, J.A. Quinolones sensitize Gram-negative bacteria to antimicrobial peptides. Antimicrob. Agents Chemother. 2006, 50, 2361-2367. [CrossRef] [PubMed]

121. Mobley, H.L.; Chippendale, G.R.; Swihart, K.G.; Welch, R.A. Cytotoxicity of the HpmA hemolysin and urease of Proteus mirabilis and Proteus vulgaris against cultured human renal proximal tubular epithelial cells. Infect. Immun. 1991, 59, 2036-2042. [PubMed] 
122. Datz, M.; Joris, B.; Azab, E.A.; Galleni, M.; van Beeumen, J.; Frère, J.M.; Martin, H.H. A common system controls the induction of very different genes. The class-A beta-lactamase of Proteus vulgaris and the Enterobacterial class-C beta-lactamase. Eur. J. Biochem. 1994, 226, 149-157. [CrossRef] [PubMed]

123. Thenmozhi, S.; Moorthy, K.; Sureshkumar, B.T.; Suresh, M. Antibiotic resistance mechanism of ESBL producing Enterobacteriaceae in clinical field: A review. Int. J. Pure App. Biosci. 2014, 2, 207-226.

124. Choi, J.G.; Kang, O.H.; Lee, Y.S.; Oh, Y.C.; Chae, H.S.; Jang, H.J.; Shin, D.W.; Kwon, D.Y. Antibacterial activity of methyl gallate isolated from Galla Rhois or carvacrol combined with nalidixic acid against nalidixic acid resistant bacteria. Molecules 2009, 14, 1773-1780. [CrossRef] [PubMed]

125. Eng, S.K.; Pusparajaha, P.; Ab Mutalibc, N.S.; Hooi-Leng Sera, H.L.; Chand, K.G.; Leea, L.H. Salmonella: A review on pathogenesis, epidemiology and antibiotic resistance. Front. Life Sci. 2015, 8, 284-293. [CrossRef]

126. Butler, T.; Sridhar, C.B.; Daga, M.K.; Pathak, K.; Pandit, R.B.; Khakhria, R.; Potkar, C.N.; Zelasky, M.T.; Johnson, R.B. Treatment of typhoid fever with azithromycin versus chloramphenicol in a randomized multicentre trial in India. J. Antimicrob. Chemother. 1999, 44, 243-250. [CrossRef] [PubMed]

127. Saavedra, M.J.; Borges, A.; Dias, C.; Aires, A.; Bennett, R.N.; Rosa, E.S.; Simões, M. Antimicrobial activity of phenolics and glucosinolate hydrolysis products and their synergy with streptomycin against pathogenic bacteria. Med. Chem. 2010, 6, 174-183. [CrossRef] [PubMed]

128. Lin, C.M.; Preston, J.F.; Wei, C.I. Antibacterial mechanism of allyl isothiocyanate. J. Food Prot. 2000, 63, 727-734. [PubMed]

129. Bosnic, T.; Softic, D.; Grujic-Vasic, J. Antimicrobial activity of some essential oils and major constituents of essential oils. Acta Med. Acad. 2006, 35, 19-22.

130. El Bouzidi, L.; Jamali, C.A.; Bekkouche, K.; Hassani, L.; Wohlmuth, H.; Leach, D.; Abbad, A. Chemical composition, antioxidant and antimicrobial activities of essential oils obtained from wild and cultivated Moroccan Thymus species. Ind. Crop. Prod. 2013, 43, 450-456. [CrossRef]

131. Nostro, A.; Sudano Roccaro, A.; Bisignano, G.; Marino, A.; Cannatelli, M.A.; Pizzimenti, F.C.; Cioni, P.L.; Procopio, F.; Blanco, A.R. Effects of oregano, carvacrol and thymol on Staphylococcus aureus and Staphylococcus epidermidis biofilms. J. Med. Microbiol. 2007, 56, 519-523. [CrossRef] [PubMed]

132. Mechergui, K.; Jaouadi, W.; Coelho, J.P.; Khouja, M.L. Effect of harvest year on production, chemical composition and antioxidant activities of essential oil of oregano (Origanum vulgare subsp. glandulosum (Desf.) Ietswaart) growing in North Africa. Ind. Crop. Prod. 2016, 90, 32-37. [CrossRef]

133. Asensio, C.M.; Grosso, N.R.; Juliani, H.R. Quality characters, chemical composition and biological activities of oregano (Origanum spp.) essential oils from central and Southern Argentina. Ind. Crop. Prod. 2015, 63, 203-213. [CrossRef]

(C) 2016 by the authors; licensee MDPI, Basel, Switzerland. This article is an open access article distributed under the terms and conditions of the Creative Commons Attribution (CC-BY) license (http://creativecommons.org/licenses/by/4.0/). 